\title{
Stable Sheaves on a Smooth Quadric Surface with Linear Hilbert Bipolynomials
}

\author{
Edoardo Ballico ${ }^{1}$ and Sukmoon Huh ${ }^{2}$ \\ ${ }^{1}$ Università di Trento, 38123 Povo, Italy \\ ${ }^{2}$ Sungkyunkwan University, Suwon 440-746, Republic of Korea \\ Correspondence should be addressed to Sukmoon Huh; sukmoonh@skku.edu
}

Received 25 August 2013; Accepted 24 October 2013; Published 11 February 2014

Academic Editors: J. Aledo and B. Nagy

Copyright (C) 2014 E. Ballico and S. Huh. This is an open access article distributed under the Creative Commons Attribution License, which permits unrestricted use, distribution, and reproduction in any medium, provided the original work is properly cited.

We investigate the moduli spaces of stable sheaves on a smooth quadric surface with linear Hilbert bipolynomial in some special cases and describe their geometry in terms of the locally free resolution of the sheaves.

\section{Introduction}

Throughout the paper, our base field is $\mathbb{C}$, the field of complex numbers.

By the work of Simpson [1], we can consider the moduli space of semistable sheaves on a smooth projective variety $X$ with a fixed Hilbert polynomial, which is itself a projective variety, and the moduli space has been studied quite intensively in the last decade for the case with linear Hilbert polynomial over projective spaces [2-5]. Our interest is on the moduli space over a smooth quadric surface.

Let $Q$ be a smooth quadric surface in $\mathbb{P}^{3}$ and let $\mathbf{M}_{Q}(\mu, \chi)$ be the moduli space of semistable sheaves on $Q$ with linear Hilbert polynomial $\chi(m)=\mu m+\chi$ with respect to the ample line bundle $\mathscr{O}_{\mathrm{Q}}(1,1)$. Unlike the case of projective spaces, this moduli space is not irreducible in general. Indeed, for a purely 1-dimensional sheaf $\mathscr{F}$ on $Q$, we can define a linear Hilbert bipolynomial $\chi_{\mathscr{F}}(x, y)$ such that

$$
\chi(\mathscr{F}(x, y))=\chi_{\mathscr{F}}(x, y)
$$

for all $(x, y) \in \mathbb{Z}^{\oplus 2}$. Then we can consider, due to [6], the moduli space $\mathbf{M}(m, n, t)$ of semistable sheaves on $Q$ with linear Hilbert bipolynomial $\chi(x, y)=m x+n y+t$. The moduli space is a projective variety with a Zariski open subset $\mathbf{M}^{\circ}(m, n, t)$ consisting of stable ones, with dimension $2 m n+1$ and the open set is nonempty if one of $m$ or $n$ is nonzero (see Proposition 7).
By its definition we have a natural decomposition

$$
\mathbf{M}_{\mathbf{Q}}(m+n, t)=\coprod_{0 \leq a \leq m+n} \mathbf{M}(a, m+n-a, t) .
$$

Thus, the moduli $\mathbf{M}(m, n, t)$ is an irreducible component of Simpson's moduli space because the bidegree function is locally constant.

If $\mathscr{F}$ is a stable sheaf in $\mathbf{M}(m, n, t)$, then its schematic support $C_{\mathscr{F}}$ is a curve of bidegree $(n, m)$ on $Q$ and so a general sheaf is a line bundle over a smooth subcurve. Thus, the moduli space can be considered as an analogue of the universal line bundle $\mathscr{P} i c_{(n, m)}^{d}$ of some fixed degree $d$ over the family of the bidegree $(n, m)$-curves in $Q$.

Now, some simple observations lead us to consider only $\mathbf{M}(m, n, t)$ with $0 \leq t \leq \operatorname{gcd}(m, n)$ due to proper twists. For small $m$ or $n$, the moduli space is very simple. Indeed, $\mathbf{M}(n, 0, t)$ is isomorphic to $\mathbb{P}^{n}$ if $t=n$ and is empty otherwise. If $m$ or $n$ is equal to 1 , say $m=1$, then it is isomorphic to $\mathbb{P}^{2 n+1}$. These descriptions are quite simple from the definition of stability condition and so the first nontrivial case happens when $(m, n)=(2,2)$. The main result of this paper is to describe the moduli spaces $\mathbf{M}(2,2, t)$ with $t=1,2$.

Theorem 1. For $\mathbf{M}_{t}=\mathbf{M}(2,2, t)$, one obtains the following:

(1) $\mathbf{M}_{1}$ is isomorphic to $\mathscr{P} i c_{(2,2)}^{1}$ and it is rational; 
(2) $\mathbf{M}_{2}$ is birational to $\mathscr{P i c}_{(2,2)}^{2}$ and it is unirational with degree 4.

In fact, we explicitly describe the sheaves in each moduli space in terms of their locally free resolution. Indeed, a sheaf $\mathscr{F}$ is in $\mathbf{M}_{1}$ if and only if it admits a resolution

$$
\begin{aligned}
0 & \longrightarrow \mathcal{O}_{Q}(-2,-1) \oplus \mathcal{O}_{Q}(-1,-2) \\
& \longrightarrow \mathcal{O}_{Q} \oplus \mathcal{O}_{Q}(-1,-1) \longrightarrow \mathscr{F} \longrightarrow 0,
\end{aligned}
$$

where the degeneracy locus of the first map is the support of $\mathscr{F}$. It enables us to identify $\mathbf{M}_{1}$ with $\mathscr{P}_{i c_{(2,2)}^{1}}$ and show its rationality.

For $\mathbf{M}_{2}$, the situation is a bit more complicated; we can classify the sheaves in $\mathbf{M}_{2}$ up to 3 types, in terms of the short exact sequences they admit, and express the moduli as the union of 3 subschemes

$$
\mathbf{M}_{2}=\mathfrak{A} \cup \mathfrak{B} \cup \mathfrak{S} .
$$

In particular, we can show that every sheaf in $\mathbf{M}_{2}$ is globally generated, from which we obtain a resolution that they admit:

$$
0 \longrightarrow \mathcal{O}_{\mathrm{Q}}(-1,-1)^{\oplus 2} \longrightarrow \mathcal{O}_{\mathrm{Q}}^{\oplus 2} \longrightarrow \mathscr{F} \longrightarrow 0 .
$$

We investigate the property of the subvarieties and the relationship between them. We also construct a map from $\mathbf{M}_{1}$ to $\mathbf{M}_{2}$, which is generically 4 to 1 and thus we obtain that $\mathbf{M}_{2}$ is unirational of degree 4 . We leave the rationality question of $\mathbf{M}_{2}$ as a conjecture.

\section{Preliminaries}

Let $Q$ be a smooth quadric surface isomorphic to $\mathbb{P} V_{1} \times \mathbb{P} V_{2}$ for 2-dimensional vector spaces $V_{1}$ and $V_{2}$, and then it is embedded into $\mathbb{P}^{3} \cong \mathbb{P} V$ by the Segre map where $V=V_{1} \otimes V_{2}$. If we denote by $f_{1}, f_{2}$ the two projections from $Q$ to each factor, then we will denote $f_{1}^{*} \mathcal{O}_{\mathbb{p}^{1}}(a) \otimes f_{2}^{*} \mathcal{O}_{\mathbb{p}^{1}}(b)$ simply by $\mathcal{O}_{\mathrm{Q}}(a, b)$. We also denote $\mathscr{E} \otimes \mathcal{O}_{\mathrm{Q}}(a, b)$ by $\mathscr{E}(a, b)$ for a coherent sheaf $\mathscr{E}$ on $Q$ and in particular the canonical sheaf $\omega_{Q}$ of $Q$ is $\sigma_{Q}(-2,-2)$.

Proposition 2. For a purely 1-dimensional sheaf $\mathscr{F}$ on $Q$, there is a bipolynomial $\chi_{\mathscr{F}}(x, y) \in \mathbb{Q}[x, y]$ of degree 1 such that

$$
\chi(\mathscr{F}(u, v))=\chi_{\mathscr{F}}(u, v)
$$

for all $(u, v) \in \mathbb{Z}^{\oplus 2}$.

Proof. Let us assume that $m t+c$ is the Hilbert polynomial of $\mathscr{F}$ with respect to the ample line bundle $\mathscr{O}_{\mathrm{Q}}(1,1)$. Let us take any $D \in\left|\mathcal{O}_{Q}(0,1)\right|, T \in\left|\mathcal{O}_{Q}(1,0)\right|$, and a smooth conic $C \in\left|\mathscr{O}_{\mathrm{Q}}(1,1)\right|$ such that neither $D, T$, nor $C$ is contained in the 1-dimensional reduced curve $\operatorname{Supp}(\mathscr{F})$.

The curves $D, T$, and $C$ induce maps $j_{D}: \mathscr{F}(t, t) \rightarrow$ $\mathscr{F}(t, t+1), j_{T}: \mathscr{F}(t, t) \rightarrow \mathscr{F}(t+1, t)$, and $j_{C}: \mathscr{F}(t, t) \rightarrow$ $\mathscr{F}(t+1, t+1)$. Since neither $D$ nor $T$ is contained in the 1 -dimensional reduced curve $\operatorname{Supp}(\mathscr{F})$, we have $j_{D} \neq 0$ and
$j_{T} \neq 0$. Since $\mathscr{F}$ is pure, we obtain that $j_{D}, j_{T}$, and $j_{C}$ are injective. Thus, there are exact sequences

$$
\begin{aligned}
& 0 \longrightarrow \mathscr{F}(t, t) \longrightarrow \mathscr{F}(t, t+1) \longrightarrow \mathscr{F}(t, t+1) \otimes \mathcal{O}_{D} \\
& \longrightarrow 0 \\
& 0 \longrightarrow \mathscr{F}(t, t) \longrightarrow \mathscr{F}(t+1, t) \longrightarrow \mathscr{F}(t+1, t) \otimes \mathcal{O}_{T} \\
& \longrightarrow 0 \\
& 0 \longrightarrow \mathscr{F}(t, t) \longrightarrow \mathscr{F}(t+1, t+1) \\
& \\
& \longrightarrow \mathscr{F}(t+1, t+1) \otimes \mathcal{O}_{C} \longrightarrow 0 .
\end{aligned}
$$

Let us set $a:=h^{0}\left(\mathscr{F}(t, t+1) \otimes \mathcal{O}_{D}\right)$ and $b:=h^{0}(\mathscr{F}(t+1, t) \otimes$ $\left.\mathcal{O}_{T}\right)$. The sheaves $\mathscr{F}(t, t+1) \otimes \mathcal{O}_{D}, \mathscr{F}(t+1, t) \otimes \mathcal{O}_{T}$, and $\mathscr{F}(t+$ $1, t+1) \otimes \mathscr{O}_{C}$ have finite supports and thus the dimensions of their cohomology $H^{0}(Q,-)$ do not change even if we twist them by a line bundle on $Q$. From (9), we get $a+b=h^{0}(\mathscr{F}(t+$ $\left.1, t+1) \otimes \mathcal{O}_{C}\right)=m$.

We claim that $\chi(\mathscr{F}(u, v))=a v+b u+c$ for all $(u, v) \in \mathbb{Z}^{\oplus 2}$. If $u=v$, then the claim is true. Now assume that $u \neq v$, say $u>$ $v$. We use $u-v$ exact sequences like $(8)$ with $\mathscr{F}(c, 0)$ instead of $\mathscr{F}$ with $0 \leq c<u-v$ to get $\chi(\mathscr{F}(u, v))=\chi(v, v)+(u-v) b$.

Definition 3. One defines the Hilbert bipolynomial $\chi_{\mathscr{F}}(x, y) \in \mathbb{Q}[x, y]$ of $\mathscr{F}$ to be a linear bipolynomial such that

$$
\chi_{\mathscr{F}}(x, y)=\chi\left(\mathscr{F} \otimes \mathcal{O}_{\mathrm{Q}}(x, y)\right)
$$

In particular, the Hilbert polynomial of $\mathscr{F}$ with respect to $\widehat{O}_{\mathrm{Q}}(1,1)$ is defined to be $\chi_{\mathscr{F}}(t)=\chi_{\mathscr{F}}(t, t)$.

We are mainly interested in the case when $\chi_{\mathscr{F}}(x, y)$ is a linear function, that is, $\chi_{\mathscr{F}}(x, y)=m x+n y+t$ for some $(m, n, t) \in \mathbb{Z}^{\oplus 3}$.

Definition 4. Let $\mathscr{F}$ be a pure sheaf of dimension 1 on $Q$ with $\chi_{\mathscr{F}}(x, y)=m x+n y+t$. The $p$-slope of $\mathscr{F}$ is defined to be $p(\mathscr{F})=t /(m+n) . \mathscr{F}$ is called semistable (stable) with respect to the ample line bundle $\mathcal{O}_{Q}(1,1)$ if

(1) $\mathscr{F}$ does not have any 0 -dimensional torsion,

(2) for any proper subsheaf $\mathscr{F}^{\prime}$, one has

$$
p\left(\mathscr{F}^{\prime}\right)=\frac{t^{\prime}}{m^{\prime}+n^{\prime}} \leq(<) \frac{t}{m+n}=p(\mathscr{F}),
$$

$$
\text { where } \chi_{\mathscr{F}^{\prime}}(x, y)=m^{\prime} x+n^{\prime} y+t^{\prime}
$$

For every semistable 1-dimensional sheaf $\mathscr{F}$ with $\chi_{\mathscr{F}}(x, y)=m x+n y+t$, let us define $C_{\mathscr{F}}:=\operatorname{Supp}(\mathscr{F})$ to be its scheme-theoretic support and then we have $C_{\mathscr{F}} \in\left|\mathcal{O}_{\mathrm{Q}}(n, m)\right|$. We often use slope stability and slope semistability instead of Gieseker stability or Gieseker semistability just to simplify the notation; they should be the same because the support is 1-dimensional, and from $m t+\chi$ and $m^{\prime} t+\chi^{\prime}$, the inequality for Hilbert and slopes $\chi / m$ the same. 
Definition 5. Let $\mathbf{M}(m, n, t)$ be the moduli space of semistable sheaves on $Q$ with linear Hilbert bipolynomial $\chi(x, y)=m x+$ $n y+t$.

We can define $\mathbf{M}(m, n, t)$ in a different way as a subvariety of $\mathbf{M}_{\mathrm{Q}, \mathbb{P}^{3}}(m+n, t)$, the moduli space of semistable sheaves on $\mathbb{P}^{3}$ with linear Hilbert polynomial $\chi(x)=m x+t$, which are $\mathcal{O}_{\mathrm{Q}^{-}}$-sheaves. To be precise, if $\mathscr{F}$ is $\mathcal{O}_{\mathrm{Q}^{-}}$sheaf, then all of its $\mathcal{O}_{\mathbb{P}^{3}-}$ subsheaves are also $\mathscr{O}_{\mathrm{Q}}$-sheaves. It implies that the notions of $p$-stability and $\mu$-stability of $\mathscr{F}$ are the same and thus $\mathbf{M}_{\mathrm{Q}, \mathbb{P}^{3}}(m+n, t)$ may be defined without using $\mathbb{P}^{3}$. Moreover, the sheaf with linear Hilbert bipolynomial $\chi(x, y)=a x+b y+$ $c$ has Hilbert polynomial $\chi(x)=(a+b) x+c$ with respect to $\mathcal{O}_{\mathrm{Q}}(1,1)$ and thus we have a natural decomposition

$$
\mathbf{M}_{\mathrm{Q}, \mathbb{P}^{3}}(m+n, t)=\coprod_{0 \leq a \leq m+n} \mathbf{M}(a, m+n-a, t) .
$$

In particular, $\mathbf{M}(m, n, t)$ is a subvariety of $\mathbf{M}_{\mathrm{Q}, \mathbb{P}^{3}}(m+n, t)$.

Remark 6. Let $\mathscr{F}$ be any purely 1-dimensional coherent sheaf on $\mathbb{P}^{3}$ with Hilbert polynomial $m x+t i$. Assume that $\mathscr{F}$ is not semistable and let

$$
0=\mathscr{F}_{0} \subset \mathscr{F}_{1} \subset \cdots \subset \mathscr{F}_{k}=\mathscr{F}
$$

be the Harder-Narasimhan filtration of $\mathscr{F}$ (see page 55 in [1]). If $\mathscr{F}$ is an $\mathcal{O}_{X}$-module, then each $\mathscr{F}_{i}$ is an $\mathcal{O}_{X}$-module because it is a subsheaf of $\mathscr{F}$. Thus the Harder-Narasimhan filtration of $\mathscr{F}$ as an $\mathcal{O}_{\mathbb{P}^{n}}$-sheaf is the same as the one as an $\mathcal{O}_{X}$-sheaf.

Proposition 7. The moduli $\mathbf{M}(m, n, t)$ is a projective and irreducible scheme. If $m n>0$, then $\mathbf{M}^{\circ}(m, n, t)$ is a Zariski dense and open subset of $\mathbf{M}(m, n, t)$ with dimension $2 m n+1$.

Proof. The first assertion follows verbatim from the proof of Proposition 2.3 and Theorem 3.1 in [6], only when the assertion in Lemma 3.3 over $Q$ holds. But it holds, using Castelnuovo-Mumford criterion with the Serre duality

$$
\begin{aligned}
H^{1}\left(\mathscr{E} x t^{1}(\mathscr{F}, \mathscr{F}(j, j))\right) & \cong \operatorname{Ext}^{2}(\mathscr{F}, \mathscr{F}(j, j)) \\
& \cong \operatorname{Hom}(\mathscr{F}(j, j), \mathscr{F}(-2,-2))^{\vee}=0
\end{aligned}
$$

for $\mathscr{F} \in \mathbf{M}(m, n, t)$ and $j \geq-1$.

For the second assertion, let us consider a map

$$
\mathbf{M}\left(m^{\prime}, n^{\prime}, t^{\prime}\right) \times \mathbf{M}\left(m^{\prime \prime}, n^{\prime \prime}, t^{\prime \prime}\right) \longrightarrow M(m, n, t)
$$

defined by sending $\left(\mathscr{F}^{\prime}, \mathscr{F}^{\prime \prime}\right)$ to $\mathscr{F}^{\prime} \oplus \mathscr{F}^{\prime \prime}$, where $m=m^{\prime}+m^{\prime \prime}$ and $n=n^{\prime}+n^{\prime \prime}$. Then the dimension of the image of this map is at least $2 m n-2 m^{\prime} n^{\prime}-2 m^{\prime \prime} n^{\prime \prime}-1$ and it is at least 1 if $m n>0$. In other words, general sheaf in $\mathbf{M}(m, n, t)$ is stable.

For any pure sheaf $\mathscr{F}$ on $Q$ with Hilbert bipolynomial $\chi_{\mathscr{F}}(x, y)=m x+n y+t$, let us define

$$
\mathscr{F}^{D}:=\mathscr{E} x t_{\mathrm{Q}}^{1}\left(\mathscr{F}, \omega_{\mathrm{Q}}\right)
$$

to be the Grothendieck dual of $\mathscr{F}$. Since $\mathscr{F}$ is pure, the natural map $\varphi_{\mathscr{F}}: \mathscr{F} \rightarrow \mathscr{F}^{D D}$ is injective. Since the support of $\mathscr{F}$ is $1-$ dimensional, $\varphi_{\mathscr{F}}$ is bijective as in Remark 4 of [4]. Moreover, the support of $\mathscr{F}^{D}$ is also 1 -dimensional and so $\chi_{\mathscr{F}^{D}}(x, y)$ is also linear. By the Serre duality, we have

$$
H^{i}\left(\mathscr{F}^{D}(c, d)\right) \cong H^{i}\left((\mathscr{F}(-c,-d))^{D}\right) \cong H^{1-i}(\mathscr{F}(-c,-d))^{\vee}
$$

for $i \in\{0,1\}$ and, in particular, we have

$$
\chi_{\mathscr{F} D}(x, y)=-\chi_{\mathscr{F}}(-x,-y)=m x+n y-t .
$$

Lemma 8. There is an isomorphism

$$
\mathbf{M}(m, n, t) \longrightarrow \mathbf{M}(m, n,-t)
$$

sending $\mathscr{F}$ to $\mathscr{F}^{D}$.

Note also that $\chi_{\mathscr{F}(d, e)}(x, y)=m x+n y+t+(m d+n e)$. Since the map

$$
\mathbf{M}(m, n, t) \longrightarrow \mathbf{M}(m, n, t+m d+n e),
$$

defined by $\mathscr{F} \mapsto \mathscr{F}(d, e)$, is an isomorphism, so we may assume that $0<t \leq \operatorname{gcd}(m, n)$.

Lemma 9. For a not necessarily integral curve $C$ in $\left|\mathcal{O}_{\mathrm{Q}}(n, m)\right|$, the sheaf $\mathrm{O}_{C}$ is semistable. If $C$ is integral, then $\hat{O}_{C}$ is stable.

Proof. We have the following sequence:

$$
0 \longrightarrow \mathcal{O}_{\mathrm{Q}}(-n,-m) \longrightarrow \mathcal{O}_{\mathrm{Q}} \longrightarrow \mathcal{O}_{\mathrm{C}} \longrightarrow 0
$$

In particular, we have $\chi_{\mathscr{O}_{C}}(x, y)=m x+n y+(m+n-m n)$ and so $p\left(\mathcal{O}_{C}\right)=1-1 /(1 / m+1 / n)$. If $C$ is integral, then $\widehat{O}_{C}$ is stable since every line bundle on an integral curve is stable. In general, $\mathscr{O}_{C}$ is semistable. Otherwise, there exists a semistable quotient sheaf $\mathscr{O}_{C} \rightarrow \mathscr{F} \rightarrow 0$ such that the Hilbert bipolynomial $\chi_{\mathscr{F}}(x, y)=m^{\prime} x+n^{\prime} y+t^{\prime}$ satisfies $m^{\prime}+n^{\prime}<m+n$ and $p(\mathscr{F})<p\left(\mathscr{O}_{C}\right)$. By induction, we get that $\mathscr{O}_{C^{\prime}}$ with $C^{\prime}:=C_{\mathscr{F}}$ is semistable and thus we have

$$
p\left(\mathscr{O}_{C^{\prime}}\right) \leq p(\mathscr{F})<p\left(\mathscr{O}_{C}\right)
$$

This is absurd since $p\left(\mathscr{O}_{C}\right)$ is a decreasing function on $m$ and $n$.

Let us assume that $m=0$, that is, $\operatorname{Hilb}_{Q}(n y+t)$ with $0<$ $t \leq n$.

Proposition 10. One has

$$
\mathbf{M}(0, n, t) \cong \begin{cases}\left(\mathbb{P}^{1}\right)^{[n]} \cong \mathbb{P}^{n} & \text { if } t=n \\ \emptyset & \text { if } 0<t<n .\end{cases}
$$

In fact, each point in $\mathrm{Hilb}_{\mathrm{Q}}(n y+n)$ corresponds to an equivalence class $\left[\mathcal{O}_{L_{1}} \oplus \cdots \oplus \mathcal{O}_{L_{n}}\right]$, where $L_{i}$ is a line in $\left|\mathscr{O}_{\mathrm{Q}}(1,0)\right|$. 
Proof. Let us assume that $t=n$ and let us choose $L \in$ $\left|\mathscr{O}_{\mathrm{Q}}(n, 0)\right|$ and then it fits into

$$
0 \longrightarrow \mathcal{O}_{\mathrm{Q}}(-n, 0) \longrightarrow \mathcal{O}_{\mathrm{Q}} \longrightarrow \mathcal{O}_{L} \longrightarrow 0 .
$$

Thus we have

$$
\begin{aligned}
\chi_{\mathcal{O}_{L}}(x, y) & =\chi_{\mathscr{O}_{\mathrm{Q}}}(x, y)-\chi_{\mathcal{O}_{\mathrm{Q}}(-n, 0)}(x, y) \\
& =(x+1)(y+1)-(x-n+1)(y+1) \\
& =n y+n .
\end{aligned}
$$

Clearly, $\mathcal{O}_{L}$ is stable. For a line $L \in\left|\mathcal{O}_{\mathrm{Q}}(1,0)\right|$, we have

$$
\chi_{\mathcal{O}_{2 L}}(x, y)=\chi_{\mathcal{O}_{L} \oplus \mathcal{O}_{L}}(x, y)=2 y+2
$$

From the sequence for $L$, we have

$$
\begin{aligned}
& 0 \longrightarrow \operatorname{Hom}\left(\mathcal{O}_{L}, \mathcal{O}_{L}\right) \longrightarrow \operatorname{Hom}\left(\mathcal{O}_{\mathrm{Q}}, \mathcal{O}_{L}\right) \\
& \quad \stackrel{f}{\longrightarrow} \operatorname{Hom}\left(\mathcal{O}_{\mathrm{Q}}(-1,0), \mathcal{O}_{L}\right) \longrightarrow \operatorname{Ext}^{1}\left(\mathcal{O}_{L}, \mathcal{O}_{L}\right) \longrightarrow 0
\end{aligned}
$$

and the map $f$ is a zero map. Thus, there exists a nontrivial extension of $\mathcal{O}_{L}$ by $\mathcal{O}_{L}$ and it is $\mathcal{O}_{2 L}$. In particular, $\mathcal{O}_{L}^{\oplus 2}$ and $\mathcal{O}_{2 L}$ represent the same point in $\operatorname{Hilb}_{Q}(2 y+2)$. In general, $\mathscr{O}_{L}^{\oplus k}$ and $\mathcal{O}_{k L}$ with $k \geq 1$ represent the same point in $\mathbf{M}(0, k, k)$. Thus, $\mathscr{O}_{L}$ with $L \in\left|\mathscr{O}_{\mathrm{Q}}(n, 0)\right|$ is strictly semistable if and only if $n \geq 2$. Conversely, let us choose a semistable sheaf $\mathscr{F}$ with $\chi_{\mathscr{F}}(x, y)=n y+n$. In particular, the schematic support $L=$ $\operatorname{Supp}(\mathscr{F})$ of $\mathscr{F}$ is in $\left|\mathscr{O}_{Q}(n, 0)\right|$. Since $\chi(\mathscr{F})=n>0$, there exists a nontrivial morphism $\mathscr{O}_{\mathrm{Q}} \rightarrow \mathscr{F}$ and it induces an injection $\mathcal{O}_{L_{1}} \rightarrow \mathscr{F}$, where $L_{1}$ is a subscheme of $L$. Here we have $L_{1} \in\left|\mathscr{O}_{\mathrm{Q}}(s, 0)\right|$ for some $s \leq n$ and so $\chi_{L_{1}}(x, y)=s x+$ $s$. Thus, the quotient $\mathscr{G}=\mathscr{F} / \mathcal{O}_{L_{1}}$ is a semistable sheaf with $\chi_{\mathscr{G}}(x, y)=(n-s) y+(n-s)$. By induction, we have $[\mathscr{G}]=\left[\mathscr{O}_{L_{2}}\right]$ with $L_{2} \in\left|\mathcal{O}_{Q}(n-s, 0)\right|$. In particular, $\mathscr{F}$ is an extension of $\mathcal{O}_{L_{2}}$ by $\mathcal{O}_{L_{1}}$ with $L_{1}+L_{2} \in\left|\mathcal{O}_{\mathrm{Q}}(n, 0)\right|$ and thus $\mathscr{F}$ is equivalent to $\mathscr{O}_{L_{1}} \oplus \mathscr{O}_{L_{2}}$.

Now, let us assume that $0<t<n$ and fix $\mathscr{F} \in \mathbf{M}(0, n, t)$ with $C:=C_{\mathscr{F}} \in\left|\mathcal{O}_{C}(n, 0)\right|$. Since $\chi(\mathscr{F})=t>0$, there is a nonzero map $f: \mathscr{O}_{Q} \rightarrow \mathscr{F}$. Since $\mathscr{F}$ is an $O_{C}$-sheaf, $f$ induces a nonzero map $h: \mathscr{O}_{C} \rightarrow \mathscr{F}$. Since $\mathscr{O}_{C}$ has slope $1>t / n$ and it is semistable, we get a contradiction. Alternatively, as in Lemma 4.10 of [6], we may first take the schematic support $T \subseteq C$ of $\operatorname{Im}(h)$ and then use an injective map $\mathcal{O}_{T} \rightarrow \mathscr{F}$ with $\mathcal{O}_{T} \in\left|\mathcal{O}_{\mathrm{Q}}\left(n^{\prime}, 0\right)\right|$ with $1 \leq n^{\prime}<n$, and thus we have $\mu\left(\mathcal{O}_{T}\right)=1$.

For the case of $m=1$, that is, $\chi_{\mathscr{F}}(x, y)=x+n y+t$, it is enough to check the case $t=1$ since $\operatorname{gcd}(1, n)=1$.

Proposition 11. $\mathbf{M}(1, n, 1)$ consists of $\mathcal{O}_{C}$ with $C \in\left|\mathcal{O}_{\mathrm{Q}}(n, 1)\right|$. In particular, one has $\mathbf{M}(1, n, 1) \cong \mathbb{P}^{2 n+1}$.

Proof. From the sequence

$$
0 \longrightarrow \mathcal{O}_{\mathrm{Q}}(-n,-1) \rightarrow \mathcal{O}_{\mathrm{Q}} \longrightarrow \mathcal{O}_{\mathrm{C}} \longrightarrow 0,
$$

we have $\chi_{\mathscr{O}_{C}}(x, y)=x+n y+1$ and $\mathscr{O}_{C}$ is semistable by Lemma 9. Conversely, let $\mathscr{F}$ be a semistable sheaf with
$\chi_{\mathscr{F}}(x, y)=x+n y+1$ and so $C:=C_{\mathscr{F}}$ is a curve in $\left|\mathcal{O}_{\mathrm{Q}}(n, 1)\right|$. Since we have $\chi(\mathscr{F})=1$, there exists a nonzero map $\mathscr{O}_{\mathrm{Q}} \rightarrow$ $\mathscr{F}$ and it induces a nonzero map $h: \mathscr{O}_{C} \rightarrow \mathscr{F}$. Note that $\operatorname{Im}(h)$ has no 0 -dimensional torsion since $\mathscr{F}$ is semistable. Since $\mathcal{O}_{C}$ is also semistable, we have

$$
p\left(\mathscr{O}_{C}\right) \leq p(\operatorname{Im}(h)) \leq p(\mathscr{F}) .
$$

The map $h$ factors through an injection $\mathcal{O}_{D} \hookrightarrow \mathscr{F}$, where $D$ is a curve contained in $C$. If $D$ is properly contained in $C$, we have $p\left(\mathcal{O}_{D}\right)>p(\mathscr{F})$ contradicting the semistability of $\mathscr{F}$ and thus we have $D=C$; that is, $h$ is an isomorphism from $\mathscr{O}_{C}$ to its image. Since $\mathscr{O}_{C}$ and $\mathscr{F}$ have the same Hilbert polynomial, we have $\mathscr{F} \cong \mathcal{O}_{C}$.

\section{Hilbert Bipolynomial $2 x+2 y+1$}

For the moduli space of semistable sheaves with linear Hilbert bipolynomial $2 x+2 y+t$, it is enough to investigate the case when $t=1,2$. Let us denote the moduli space $\mathbf{M}(2,2, t)$ by $\mathbf{M}_{t}$.

Proposition 12. The moduli space $\mathbf{M}_{1}$ consists of the unique nontrivial extensions $\mathscr{F}$ of $\mathcal{O}_{P}$ by $\mathcal{O}_{C}$ for each curve $C \in$ $\left|\mathcal{O}_{\mathrm{Q}}(2,2)\right|$ and a point $P \in C$, and one also has $h^{0}(\mathscr{F})=1$.

Proof. Since $\chi(\mathscr{F})=1$, there is a nonzero map $\mathcal{O}_{\mathrm{Q}} \rightarrow \mathscr{F}$, inducing a nonzero map $h: \mathscr{O}_{C} \rightarrow \mathscr{F}$, where $C:=C_{\mathscr{F}} \in$ $\left|\mathcal{O}_{\mathrm{Q}}(2,2)\right|$. Since $\chi_{\mathscr{O}_{C}}(x, y)=2 x+2 y$, we have $p\left(\mathcal{O}_{C}\right)=$ $0<1 / 4=p(\mathscr{F})$. The map $h$ factors through an injection $\mathcal{O}_{D} \hookrightarrow \mathscr{F}$, where $D$ is a curve contained in $C$. If $D$ is properly contained in $C$, we have $p\left(\mathscr{O}_{D}\right)>p(\mathscr{F})$ contradicting to the semistability of $\mathscr{F}$ and thus we have $D=C$; that is, $h$ is an isomorphism from $\mathscr{O}_{C}$ to its image, that is, we have

$$
0 \longrightarrow \mathcal{O}_{\mathrm{C}} \longrightarrow \mathscr{F} \longrightarrow \mathscr{G} \longrightarrow 0
$$

where $\chi_{\mathscr{G}}(x, y)=1$. In particular, we have $\mathscr{G} \cong \mathcal{O}_{P}$, the skyscraper sheaf supported on a point $P \in C$. Since $\mathscr{F}$ has no 0 -dimensional torsion, the sequence does not split. Note that $\operatorname{Ext}^{1}\left(\mathcal{O}_{P}, \mathcal{O}_{Q}\right) \cong H^{1}\left(\mathcal{O}_{P}\right)^{\vee}=0$, and thus from the sequence of $C$ we have

$$
\begin{aligned}
& 0 \longrightarrow \operatorname{Ext}^{1}\left(\mathcal{O}_{P}, \mathcal{O}_{C}\right) \longrightarrow \operatorname{Ext}^{2}\left(\mathcal{O}_{P}, \mathcal{O}_{\mathrm{Q}}(-2,-2)\right) \\
& \stackrel{s}{\longrightarrow} \operatorname{Ext}^{2}\left(\mathcal{O}_{P}, \mathcal{O}_{\mathrm{Q}}\right) .
\end{aligned}
$$

Here, the map $s$ is the transpose of $\operatorname{Hom}\left(\mathcal{O}_{Q}(2,2), \mathcal{O}_{P}\right) \rightarrow$ $\operatorname{Hom}\left(\mathcal{O}_{\mathrm{Q}}, \mathcal{O}_{P}\right)$ which is given by the multiplication by the defining equation of $C$. Since $P$ is a point on $C$, the map $s$ is a zero map. In particular, the dimension of $\operatorname{Ext}^{1}\left(\mathcal{O}_{P}, \mathcal{O}_{C}\right)$ is 1 and so $\mathscr{F}$ corresponds to a unique nontrivial extension

$$
0 \longrightarrow \mathcal{O}_{C} \longrightarrow \mathscr{F} \longrightarrow \mathcal{O}_{P} \longrightarrow 0
$$

From the sequence $(32)$, we have $h^{0}(\mathscr{F}) \leq 2$ and that $h^{0}(\mathscr{F})=$ 1 if and only if no injective map $\mathscr{O}_{C} \rightarrow \mathscr{F}$ is an isomorphism at $P$. This is certainly true if $\mathscr{F}$ is not locally free of rank 1 at $P$. Note that $\mathscr{F}$ is a line bundle at each point of $C \backslash\{P\}$ and thus it is sufficient to prove $h^{0}(\mathscr{F})=1$ when $\mathscr{F}$ is a line bundle on 
the curve $C$. In this case the nonexistence of a section of $\mathscr{F}$ that does not vanish at $P$ is equivalent to the nonsplitting of (32). Thus, we have $h^{0}(\mathscr{F})=0$ and so the point $P$ is uniquely determined by $\mathscr{F}$.

Conversely, let us assume that $\mathscr{F}$ is a nontrivial extension of $\mathcal{O}_{P}$ by $\mathscr{O}_{C}$, where $P$ is a point on $C$. If $\mathscr{F}$ is not semistable, then there exists a subsheaf $\mathscr{K} \subset \mathscr{F}$ with $p(\mathscr{K})>p(\mathscr{F})=1 / 4$ and so we have $\chi_{\mathscr{K}}(x, y)=m^{\prime} x+n^{\prime} y+t^{\prime}$ with $\left(m^{\prime}, n^{\prime}\right) \leq(2,2)$ and $t^{\prime} \geq 1$. If the composite $s: \mathscr{K} \rightarrow \mathscr{F} \rightarrow \mathcal{O}_{P}$ is a zero map, then we have an injection $\mathscr{K} \hookrightarrow \mathcal{O}_{C}$, contradicting the semistability of $\mathscr{O}_{C}$. Thus, the composite is surjective and so we have the following diagram:

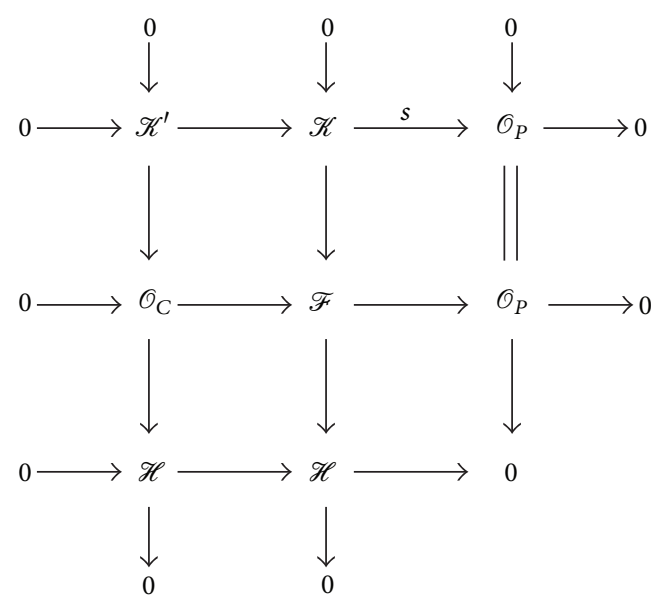

Here, $\mathscr{K}^{\prime}$ is the kernel of the map $s$ and $\mathscr{H}$ is the quotient $\mathscr{F} / \mathscr{K}$. Since $\chi_{\mathscr{K}^{\prime}}(x, y)=m^{\prime} x+n^{\prime} y+\left(t^{\prime}-1\right)$ and $\mathscr{O}_{C}$ is semistable, we have $t^{\prime}=1$ and thus $\chi_{\mathscr{H}}(x, y)=\left(2-m^{\prime}\right) x+$ $\left(2-n^{\prime}\right) y$ with no constant term. Since $\mathscr{H}$ is the quotient of $\mathcal{O}_{C}$, it must be $\mathcal{O}_{T}$ for some curve $T$ contained in $C$. But no such curves have the Hilbert polynomials with no constant term. Hence $\mathscr{F}$ is semistable.

Remark 13. There is no strictly semistable sheaf in $\mathbf{M}_{1}$. Let us assume the existence of a polystable sheaf $\mathscr{F}=\mathscr{F}_{1} \oplus \cdots \oplus \mathscr{F}_{s}$ with $s \geq 2$. We have $\chi(\mathscr{F})=1=\chi\left(\mathscr{F}_{1}\right)+\cdots+\chi\left(\mathscr{F}_{s}\right)$. If we let $\chi_{\mathscr{F}_{i}}(x, y)=a_{i} x+b_{i} y+c_{i}$, then we have

$$
c_{1}+\cdots+c_{s}=1, \quad \frac{c_{i}}{a_{i}+b_{i}}=\frac{1}{2}, \quad \forall i .
$$

It implies that $c_{i}>0$ for all $i$ and thus we have $s=1$, a contradiction.

Proposition 14. A sheaf $\mathscr{F}$ is in $\mathbf{M}_{1}$ if and only if it admits the following resolution:

$$
0 \longrightarrow \mathscr{A} \stackrel{\varphi}{\longrightarrow} \mathcal{O}_{\mathrm{Q}} \oplus \mathcal{O}_{\mathrm{Q}}(-1,-1) \stackrel{\psi}{\longrightarrow} \mathscr{F} \longrightarrow 0,
$$

where $\mathscr{A}:=\mathcal{O}_{\mathrm{Q}}(-2,-1) \oplus \mathcal{O}_{\mathrm{Q}}(-1,-2)$ and $\varphi=\left(\begin{array}{ll}h_{1} & l_{1} \\ h_{2} & l_{2}\end{array}\right)$. Here, $f:=h_{1} l_{2}-h_{2} l_{1}$ is a defining equation of $C_{\mathscr{F}}$.

Proof. Note that $h^{0}(\mathscr{F})=1$ and so $h^{1}(\mathscr{F})=0$. If $\mathscr{F}$ admits the sequence (32), then $\mathscr{F}$ is globally generated outside $P$ and so is $\mathscr{F}(1,1)$. Take any $A \in\left|\mathscr{O}_{\mathrm{Q}}(1,1)\right|$ which is not contained in $C$ and with $P \notin A$. The multiplication by an equation of $A$ gives an exact sequence

$$
\left.0 \longrightarrow \mathscr{F} \longrightarrow \mathscr{F}(1,1) \longrightarrow \mathscr{F}(1,1)\right|_{A} \longrightarrow 0,
$$

where $\operatorname{deg}\left(\left.\mathscr{F}(1,1)\right|_{A}\right)=\operatorname{deg}(A \cap C)=4$. Thus we have $h^{1}(\mathscr{F}(1,1))=0$ and $h^{0}(\mathscr{F}(1,1))=5$. Together with the exact sequence (32) tensored by $\mathcal{O}_{\mathrm{Q}}(1,1)$, we obtain that $\mathscr{F}(1,1)$ is globally generated at $P$ and so we have a surjection

$$
\psi: \mathcal{O}_{\mathrm{Q}} \oplus \mathcal{O}_{\mathrm{Q}}(-1,-1) \longrightarrow \mathscr{F} \longrightarrow 0 .
$$

Let us set $\mathscr{H}:=\operatorname{ker}(\psi)$ and then $\mathscr{H}$ is a torsion-free sheaf of rank 2 on $Q$ with $c_{1}=(-3,-3)$. By Theorem 19.9 in [7], the sheaf $\mathscr{H}$ is locally free. Note that $\chi_{\mathscr{H}(1,2)}(x, y)=2 x y+3 x+$ $y+1$. Thus, we have $h^{0}(\mathscr{H}(1,2))>0$ and so we have an exact sequence

$$
0 \longrightarrow \mathcal{O}_{\mathrm{Q}}(a, b) \longrightarrow \mathscr{H}(1,2) \longrightarrow \mathscr{I}_{Z}(-1-a, 1-b) \longrightarrow 0,
$$

where $Z$ is a 0 -dimensional subscheme of $Q$ and $(a, b) \epsilon$ $\{(0,0),(0,1)\}$. If $(a, b)=(0,1)$, then we have $\chi_{\mathscr{I}_{Z}(-1,0)}(x, y)=$ $x y+x+1$ and it is absurd since $\chi_{\mathcal{O}_{\mathrm{Q}}(-1,0)}(x, y)=x y+x$. Thus, we have $(a, b)=(0,0)$ and $Z=\emptyset$. Since $\operatorname{Ext}^{1}\left(\mathscr{O}_{\mathrm{Q}}(-1,1), \mathscr{O}_{\mathrm{Q}}\right)=$ 0 , we have $\mathscr{H}(1,2) \cong \mathcal{O}_{\mathrm{Q}} \oplus \mathcal{O}_{\mathrm{Q}}(-1,1)$ and the sequence $(35)$. Note that the map $\varphi: \mathscr{H} \rightarrow \mathcal{O}_{\mathrm{Q}} \oplus \mathcal{O}_{\mathrm{Q}}(-1,-1)$ is given by $\left(\begin{array}{ll}h_{1} & l_{1} \\ h_{2} & l_{2}\end{array}\right)$, where $f:=h_{1} l_{2}-h_{2} l_{1}$ is a defining equation of $C=C_{\mathscr{F}}$.

The converse is trivial.

Remark 15. Using the proof of Lemma 5.3 in [2], we can obtain the same assertion of Proposition 14. Similarly, we also obtain that $\mathscr{F}(1,0)$ is globally generated and so a surjection $\varphi^{\prime}: \mathscr{O}_{Q} \oplus \mathcal{O}_{Q}(-1,0) \rightarrow \mathscr{F}$. In this case, $\operatorname{ker}\left(\varphi^{\prime}\right)$ is no longer a direct sum of two line bundles.

Let us define a vector space $W$ to be

$$
W:=\operatorname{Hom}\left(\mathscr{A}, \mathcal{O}_{\mathrm{Q}} \oplus \mathcal{O}_{\mathrm{Q}}(-1,-1)\right)
$$

and $W^{0} \subset W$ to be the set of $\varphi \in W$ such that $h_{1} l_{2}-h_{2} l_{1} \neq 0$. Then we have a surjective morphism

$$
\pi: W^{0} \longrightarrow M_{1} \text {. }
$$

Let us choose $\varphi_{1}, \varphi_{2} \in W^{0}$ with $\pi\left(\varphi_{1}\right)=\pi\left(\varphi_{2}\right)$; that is, we have the following diagram:

$$
\begin{aligned}
0 \longrightarrow \mathscr{A} \stackrel{\varphi_{1}}{\longrightarrow} \mathcal{O}_{\mathrm{Q}} \oplus \mathcal{O}_{\mathrm{Q}}(-1,-1) \stackrel{\psi_{1}}{\longrightarrow} \mathscr{F} \longrightarrow 0 \\
\downarrow \\
0 \longrightarrow \mathscr{A} \stackrel{\varphi_{2}}{\longrightarrow} \mathcal{O}_{\mathrm{Q}} \oplus \mathcal{O}_{\mathrm{Q}}(-1,-1) \stackrel{\psi_{2}}{\longrightarrow} \mathscr{F} \longrightarrow 0,
\end{aligned}
$$

where $f$ is an isomorphism. Since $\operatorname{Ext}^{1}\left(\mathcal{O}_{\mathrm{Q}} \oplus\right.$ $\left.\mathscr{O}_{\mathrm{Q}}(-1,-1), \mathscr{A}\right)=0$, we have a map $f_{1} \in \operatorname{End}\left(\mathscr{O}_{\mathrm{Q}} \oplus\right.$ $\left.\mathcal{O}_{\mathrm{Q}}(-1,-1)\right)$ associated with $f$. Note that $f_{1}$ is given by $\left(\begin{array}{ll}a & 0 \\ z & b\end{array}\right)$, where $a, b \in \mathbb{C}^{\times}$and $z \in H^{0}\left(\mathcal{O}_{\mathrm{Q}}(1,1)\right)$. Similarly, we have 
a map $f_{2}: \mathscr{A} \rightarrow \mathscr{A}$ which is $\left(\begin{array}{cc}c_{1} & 0 \\ 0 & c_{2}\end{array}\right)$, where $c_{1}, c_{2} \in \mathbb{C}^{\times}$. In particular, we have

$$
\varphi_{2}=\left(\begin{array}{cc}
c_{1} & 0 \\
0 & c_{2}
\end{array}\right)^{-1} \varphi_{1}\left(\begin{array}{ll}
a & 0 \\
z & b
\end{array}\right)
$$

In this equation, we can assume that $c_{1}=1$. In other words, $\pi\left(\varphi_{1}\right)=\pi\left(\varphi_{2}\right)$ if and only if $\varphi_{1}$ and $\varphi_{2}$ are in the same orbit in $W^{0}$ under the action by

$$
\begin{gathered}
\mathbf{G}:=\frac{\left(\operatorname{Aut}(\mathscr{A}) \times \operatorname{Aut}\left(\mathcal{O}_{\mathrm{Q}} \oplus \mathcal{O}_{\mathrm{Q}}(-1,-1)\right)\right)}{\mathbb{C}^{\times}} \\
=\left\{\left(\left(\begin{array}{ll}
1 & 0 \\
0 & c
\end{array}\right),\left(\begin{array}{ll}
a & 0 \\
z & b
\end{array}\right)\right) \mid a, b, c \in \mathbb{C}^{\times},\right. \\
\left.z \in H^{0}\left(\mathcal{O}_{\mathrm{Q}}(1,1)\right)\right\} .
\end{gathered}
$$

Theorem 16. $\pi: W^{0} \rightarrow \mathbf{M}_{1}$ is a geometric quotient map by the action of $\mathbf{G}$. In particular, one has $\mathbf{M}_{1} \cong W^{0} / \mathbf{G}$ and so $\mathbf{M}_{1}$ is isomorphic to $\mathscr{P i c}_{(2,2)}^{1}$.

Proof. To get the assertion it suffices to prove that it has local sections as in Lemma 5.1 and Theorem 5.5 in [3].

Since every element of $\mathbf{M}_{1}$ is stable, $\mathbf{M}_{1}$ has a universal family $\mathbb{H}_{1}$ on $\mathbf{M}_{1} \times \mathbb{P}^{2}$ (see page 180 of [8] or Theorem 4.6 .5 of [9]). Since every semistable sheaf with bipolinomial $2 x+2 y+5$ is of the form $\mathscr{F}(1,1)$ for a unique $\mathscr{F} \in \mathbf{M}_{1}$, we also have a universal family $\mathbb{H}_{5}$ on $\mathbf{M}_{5} \times \mathbb{P}^{2}$ with $\mathscr{F}(1,1)$ as the fibre. Since $h^{0}(\mathscr{F})=1$ and $h^{1}(\mathscr{F})=0$ for all $\mathscr{F} \in \mathbf{M}_{1}$, the base change theorem gives that $u_{*}\left(\mathbb{H}_{1}\right)$ is a line bundle on $\mathbf{M}_{1}$, where $u: \mathbf{M}_{1} \times \mathbb{P}^{2} \rightarrow M_{1}$ is the first projection. Since $h^{0}(\mathscr{F}(1,1))=5$ and $h^{1}(\mathscr{F}(1,1))=0$ for all $\mathscr{F} \in \mathbf{M}_{1}$, the base change theorem gives that $v_{*}\left(\mathbb{H}_{5}\right)$ is a vector bundle of rank 5 on $\mathbf{M}_{1}$ by identifying $\mathbf{M}_{5}$ with $\mathbf{M}_{1}$, where $v: \mathbf{M}_{5} \times \mathbb{P}^{2} \rightarrow M_{5}$. For a fixed $\mathscr{F} \in \mathbf{M}_{1}$ and a matrix $\varphi \in \pi^{-1}(\mathscr{F})$, let us write $\varphi=\left(\begin{array}{ll}h_{1} & l_{1} \\ h_{2} & l_{2}\end{array}\right)$, where $f:=h_{1} l_{2}-h_{2} l_{1}$ is a defining equation of $C_{\mathscr{F}}$. Take an open neighborhood $U$ of $\mathscr{F}$ in $\mathbf{M}_{1}$ over which $u_{*}\left(\mathbb{H}_{1}\right)$ and $v_{*}\left(\mathbb{H}_{5}\right)$ are trivial. The matrix $\varphi$ was constructed starting with a section $\sigma$ of $\mathscr{F}(1,1)$ which spans $\mathscr{F}(1,1)$ together with the twist $\sigma^{\prime}$ of a nonzero section of $\mathscr{F}$. Since $\left.u_{*}\left(\mathbb{H}_{1}\right)\right|_{U}$ and $\left.v_{*}\left(\mathbb{H}_{5}\right)\right|_{U}$ are trivial, there are maps $e_{1}: \mathcal{O}_{U} \rightarrow \mathcal{O}_{U}$ and $e_{2}: \mathscr{O}_{U} \rightarrow \mathcal{O}_{U}^{\oplus 5}$ with $e_{1}(\mathscr{F})=\sigma^{\prime}$ and $e_{2}(\mathscr{F})=\sigma$. Since $\sigma^{\prime}$ and $\sigma$ span $\mathscr{F}$, there is a neighborhood $V$ of $\mathscr{F}$ in $U$ such that the sections $e_{1}(\mathscr{G})$ and $e_{2}(\mathscr{G})$ span every $\mathscr{G} \in V$. The construction of $\varphi$ gives that $e_{1}$ and $e_{2}$ induce a section of $\pi$ in a neighborhood of $\varphi$ whose image by $\pi$ is $V$.

As an automatic consequence, we obtain that $\mathbf{M}_{1}$ is irreducible and unirational. In fact, we can prove more.

\section{Theorem 17. $\mathbf{M}_{1}$ is rational.}

Proof. Let $\Delta \subset Q \times Q$ be the diagonal and denote its ideal sheaf by $\mathscr{I}_{\Delta}$. Denoting by $p_{1}$ and $p_{2}$ the projection from $Q \times Q$ to each factor, let us define a sheaf $\mathscr{U}$ to be $\left.p_{1}^{*} \mathscr{O}_{\mathrm{Q}}(2,2)\right) \otimes \mathscr{I}_{\Delta}$ on $Q \times Q$. For each point $P \in Q$, we have $\left.\mathscr{U}\right|_{Q \times\{P\}} \cong \mathscr{I}_{P}(2,2)$. Thus, we have $h^{1}\left(\left.\mathcal{U}\right|_{\mathrm{Q} \times\{P\}}\right)=0$ and so $p_{2 *} \mathcal{U}$ is a vector bundle of rank 8 on $Q$ since $h^{0}\left(\left.\mathcal{U}\right|_{Q \times\{P\}}\right)=8$. Let us consider the projective bundle

$$
\mathscr{Z}=\mathbb{P}\left(p_{2 *} \mathcal{U}\right) \longrightarrow Q \text {. }
$$

By its definition, the fibre of $\mathscr{Z}$ over a point $P \in Q$ is the set of curves of type $(2,2)$ on $Q$ passing through $P$ and so there is a natural map from $\mathscr{Z}$ to $\mathbb{P} H^{0}\left(\mathcal{O}_{\mathrm{Q}}(2,2)\right) \cong \mathbb{P}^{8}$. In other words, $\mathscr{Z}$ is the universal curve of type $(2,2)$ on $Q$ and it is isomorphic to $\mathbf{M}_{1}$. Since $\mathscr{Z}$ is locally trivial over $Q$, it is rational.

\section{Hilbert Bipolynomial $2 x+2 y+2$}

Lemma 18. Any sheaf $\mathscr{F} \in \mathbf{M}_{2}$ admits one of the following types:

$$
\begin{aligned}
& \text { (A) } 0 \rightarrow \mathcal{O}_{C} \rightarrow \mathscr{F} \rightarrow \eta \rightarrow 0 \text {, where } \eta \text { is a skyscraper } \\
& \mathcal{O}_{C^{-} \text {sheaf with degree } 2,} \\
& \text { (B) } 0 \rightarrow \mathcal{O}_{T_{1}} \rightarrow \mathscr{F} \rightarrow \mathcal{O}_{T_{2}} \rightarrow 0 \text { with } T_{1}, T_{2} \in \\
& \left|\mathcal{O}_{\mathrm{Q}}(1,1)\right|, \\
& \text { (C) } 0 \rightarrow \mathcal{O}_{T_{1}} \rightarrow \mathscr{F} \rightarrow \mathcal{O}_{T_{2}} \rightarrow 0 \text {, where } T_{1} \in\left|\mathcal{O}_{\mathrm{Q}}(a, b)\right| \\
& \text { and } T_{2} \in\left|\mathcal{O}_{\mathrm{Q}}(2-a, 2-b)\right| \text { with }(a, b) \in\{(1,2),(2,1)\} .
\end{aligned}
$$

Proof. Since $\chi(\mathscr{F})=2$, we have $h^{0}(\mathscr{F}) \geq 2$. Thus, there exists a nonzero map $\mathscr{O}_{\mathrm{Q}} \rightarrow \mathscr{F}$ and it induces a nonzero map $h:$ $\mathcal{O}_{C} \rightarrow \mathscr{F}$, where $C:=C_{\mathscr{F}} \in\left|\mathcal{O}_{\mathrm{Q}}(2,2)\right|$. The map $h$ factors through an injection $\mathcal{O}_{T_{1}} \hookrightarrow \mathscr{F}$ where $T_{1}$ is a curve contained in $C$ :

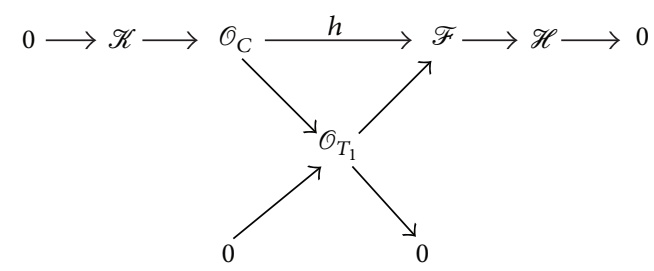

If we have $T_{1}=C$, that is the map $h$ is an isomorphism from $\mathcal{O}_{\mathrm{C}}$ to its image in $\mathscr{F}$, then its cokernel $\mathscr{H}$ is the skyscraper sheaf supported on two points, say $P_{1}, P_{2} \in C$. Thus we have the sequence

$$
0 \longrightarrow \mathcal{O}_{C} \longrightarrow \mathscr{F} \longrightarrow \eta \longrightarrow 0 \text {. }
$$

Let us assume that $T_{1}$ is properly contained in $C$ and then we obtain that $T_{1}$ has bidegree $(1,1),(1,2)$, or $(2,1)$ since $p\left(\mathcal{O}_{T_{1}}\right) \leq p(\mathscr{F})=1 / 2$ and $\mathscr{F}$ is semistable. Let $T_{2} \subset Q$ be the only curve such that $T_{1}+T_{2}=C$. Let $\mathscr{H}^{\prime}$ be the quotient of $\mathscr{H}$ by its torsion $\tau$, that is, $\mathscr{H}^{\prime}:=\mathscr{H}^{D D}$.

First, assume $T_{1} \in\left|\mathcal{O}_{\mathrm{Q}}(1,1)\right|$ and so we have $\chi_{\mathscr{H}^{\prime}}(x, y)=$ $x+y+1-\operatorname{deg}(\tau)$. Since $\mathscr{F}$ is semistable, we get $\tau=0$. Since every quotient of $\mathscr{F}$ has the slope at least $1 / 2$, the same is true for $\mathscr{H}$. Thus, $\mathscr{H}$ is semistable and Proposition 11 gives $\mathscr{H} \cong$ $\mathcal{O}_{T_{2}}$.

Now, without loss of generality, let us assume that $T_{1} \in$ $\left|\mathcal{O}_{\mathrm{Q}}(1,2)\right|$, that is, $\chi_{\mathcal{O}_{T_{1}}}(x, y)=2 x+y+1$ and so we have $\chi_{\mathscr{H}}(x, y)=y+1$. If $\mathscr{H}$ has 0 -dimensional torsion $\mathscr{T}$ with length $k \geq 1$, then the quotient $\mathscr{H} / \mathscr{T}$ is a quotient of $\mathscr{F}$ with 
the $p$-slope $1-k \leq 0$, contradicting the semistability of $\mathscr{F}$. Thus $\mathscr{H}$ has no 0 -dimensional torsion and so we have $\mathscr{H} \cong$ $\mathcal{O}_{T_{2}}$ for a curve $T_{2} \in\left|\mathcal{O}_{Q}(1,0)\right|$ with $C=T_{1}+T_{2}$.

Corollary 19. Every sheaf in $\mathbf{M}_{2}$ is globally generated.

Proof. Let us take $\mathscr{F} \in \mathbf{M}_{2}$ and then there is no nonzero map $\mathscr{F} \rightarrow \mathcal{O}_{C} \cong \omega_{C}$ since $\mathscr{F}$ is semistable. Thus we have $h^{1}(\mathscr{F})=$ 0 and so $h^{0}(\mathscr{F})=2$. It is clear that $\mathscr{F}$ of types (B) and (C) is globally generated and so we may assume that $\mathscr{F}$ is of type (A), but neither of (B) nor of (C).

Let $\mathscr{H} \subseteq \mathscr{F}$ be the image of the evaluation map $H^{0}(\mathscr{F}) \otimes$ $\mathcal{O}_{\mathrm{Q}} \rightarrow \mathscr{F}$ and then $\mathscr{H}$ is pure. Assume that $\mathscr{H} \neq \mathscr{F}$. Since $\mathscr{F}$ is of type (A), it is globally generated outside at most two points of $C_{\text {red }}$. In particular, we have $\chi_{\mathscr{H}}(x, y)=2 x+2 y+c$ with $c \leq 1$ and $\operatorname{deg}(\mathscr{F} / \mathscr{H})=2$. Since $h^{0}(\mathscr{H})=h^{0}(\mathscr{F})=2$, we have $h^{1}(\mathscr{H})=2-c$. Note that every nonzero section of $\mathscr{H}$ vanishes at finitely many points since $\mathscr{F}$ is neither of types (B) nor $(\mathrm{C})$. Since $h^{0}\left(\mathscr{O}_{C}\right)<h^{0}(\mathscr{H})$, we have $\mathscr{H} \neq \mathscr{O}_{C}$ and $c=1$. A nonzero section of $\mathscr{H}$ induces an exact sequence

$$
0 \longrightarrow \mathcal{O}_{\mathrm{C}} \longrightarrow \mathscr{H} \longrightarrow \mathscr{G} \longrightarrow 0,
$$

where $\mathscr{G} \cong \mathcal{O}_{P}$ for some $P \in C_{\text {red }}$. Since $\mathscr{H}$ is pure, this exact sequence does not split. As in the proof of Proposition 12, we get a contradiction. Thus, we have $\mathscr{H}=\mathscr{F}$ and so $\mathscr{F}$ is globally generated.

Lemma 20. $\mathscr{F}$ is a sheaf in $\mathbf{M}_{2}$ if and only if it admits a sequence

$$
0 \longrightarrow \mathcal{O}_{\mathrm{Q}}(-1,-1)^{\oplus 2} \stackrel{\varphi}{\longrightarrow} \mathcal{O}_{\mathrm{Q}}^{\oplus 2} \longrightarrow \mathscr{F} \longrightarrow 0,
$$

where $\varphi=\left(\begin{array}{ll}z_{11} & z_{12} \\ z_{21} & z_{22}\end{array}\right), z_{i j} \in H^{0}\left(\mathcal{O}_{Q}(1,1)\right)$ such that $f:=z_{11} z_{22}-$ $z_{12} z_{21}$ is a defining equation of $C_{\mathscr{F}}$.

Proof. Let $\mathscr{F} \in \mathbf{M}_{2}$ be a sheaf of type (A) and then it is globally generated by Corollary 19. Since $h^{0}(\mathscr{F})=2$, we have a surjection

$$
\psi: \mathcal{O}_{\mathrm{Q}}^{\oplus 2} \longrightarrow \mathscr{F} \longrightarrow 0 .
$$

Let us set $\mathscr{H}:=\operatorname{ker}(\psi)$ and then it is a torsion-free sheaf of rank 2 on $Q$ with $c_{1}=(-2,-2)$. By Theorem 19.9 in [7], $\mathscr{H}$ is locally free. Note that $h^{0}(\mathscr{H}(1,1))=2$. From the sequence

$$
0 \longrightarrow \mathscr{H}(1,0) \longrightarrow \mathcal{O}_{\mathrm{Q}}(1,0)^{\oplus 2} \longrightarrow \mathscr{F}(1,0) \longrightarrow 0
$$

we obtain that the map $H^{0}\left(\mathcal{O}_{\mathrm{Q}}(1,0)^{\oplus 2}\right) \rightarrow H^{0}(\mathscr{F}(1,0))$ is an isomorphism and so $h^{1}(\mathscr{H}(1,0))=0$. Similarly, we have $h^{1}(\mathscr{H}(0,1))=0$ and $h^{2}(\mathscr{H})=h^{1}(\mathscr{F})=0$. By Remark 2.3 in [10], we obtain that $\mathscr{H}(1,1)$ is globally generated. Since $c_{1}(\mathscr{H}(1,1))=0$ or $h^{0}(\mathscr{H}(1,1))=2$, we have $\mathscr{H} \cong$ $\mathcal{O}_{\mathrm{Q}}(-1,-1)^{\oplus 2}$ and the resolution (48). The cases of the other types also work verbatim.

Definition 21. Let us define a subscheme $\mathfrak{A} \subset \mathbf{M}_{2}$ as follows:

$$
\begin{gathered}
\mathfrak{A}:=\left\{\mathscr{F} \in \mathbf{M}_{2} \mid \mathscr{F}\right. \text { admits a nontrivial } \\
\text { extension of type }(\mathrm{A})\} .
\end{gathered}
$$

Similarly, we define $\mathfrak{B}$ and $\mathfrak{C}$ for the semistable sheaves of types (B) and (C), respectively. In particular, we have

$$
\mathbf{M}_{2}=\mathfrak{A} \cup \mathfrak{B} \cup \mathfrak{C} .
$$

Lemma 22. The sheaves $\mathscr{F}$ of type $(B)$ are strictly semistable. In particular, they are contained in $\mathfrak{B}$.

Proof. It is enough to check the semistability of $\mathscr{F}$. Let $\mathscr{K}$ be a subsheaf of $\mathscr{F}$ with $p(\mathscr{K})>1 / 2$ and the quotient sheaf $\mathscr{H}:=\mathscr{F} / \mathscr{K}$. If the composite map $s: \mathscr{K} \hookrightarrow \mathscr{F} \rightarrow \mathcal{O}_{T_{2}}$ is a zero map, then $\mathscr{K}$ is a subsheaf of $\mathcal{O}_{T_{1}}$, contradicting the semistability of $\mathcal{O}_{T_{1}}$. The sheaf $\operatorname{Im}(s)$ is a subsheaf of $\mathcal{O}_{T_{2}}$ and so we have $p(\operatorname{Im}(s)) \leq 1 / 2$. Similarly, the sheaf $\operatorname{ker}(s)$ is a subsheaf of $\mathcal{O}_{T_{1}}$ and so $p(\operatorname{ker}(s)) \leq 1 / 2$. From the exact sequence

$$
0 \longrightarrow \operatorname{ker}(s) \longrightarrow \mathscr{K} \stackrel{s}{\longrightarrow} \operatorname{Im}(s) \longrightarrow 0
$$

we have $p(\mathscr{K}) \leq 1 / 2$, a contradiction.

Let us denote by $\partial \mathbf{M}_{2}$ the closed subscheme of $\mathbf{M}_{2}$, consisting of the strictly semistable sheaves.

Corollary 23. One has

$$
\partial \mathbf{M}_{2}=\mathfrak{B} \cong \frac{\left(\mathbb{P}^{3} \times \mathbb{P}^{3}\right)}{\mathfrak{S}_{2}},
$$

where $\mathfrak{S}_{2}$ is the permutation group of order 2 . In particular, $\partial \mathbf{M}_{2}$ is a rational variety.

Proof. Obviously, we have $\mathfrak{B} \subset \partial \mathbf{M}_{2}$. Let $\mathscr{F}$ be a strictly semistable sheaf and so it has a proper quotient sheaf $\mathscr{H}$ with $p(\mathscr{H})=1 / 2$. From the semistability of $\mathscr{F}, \mathscr{H}$ has no 0 -dimensional torsion. From the equality $p(\mathscr{F})=p(\mathscr{H})$, we obtain that $\mathscr{H}$ is also semistable. Since $p(\mathscr{H})=1 / 2$, the Hilbert bipolynomial of $\mathscr{H}$ is either $2 x+1,2 y+1$ or $x+y+1$. The first 2 cases cannot happen due to Proposition 10 . Thus, we have $\chi_{\mathscr{H}}(x, y)=x+y+1$ and so $\mathscr{H} \cong \mathcal{O}_{T_{2}}$ with $T_{2} \in\left|\mathcal{O}_{Q}(1,1)\right|$ and $T_{2} \subset C_{\mathscr{F}}$ by Proposition 11. If $\mathscr{K}^{2}$ is the kernel of the quotient map $\mathscr{H} \rightarrow \mathscr{H}$, then its p-slope is again $1 / 2$ and so $\mathscr{K}$ is semistable. Similarly as before, we have $\mathscr{K} \cong \mathcal{O}_{T_{1}}$ with $T_{1} \in\left|\mathcal{O}_{\mathrm{Q}}(1,1)\right|$ and $C_{\mathscr{F}}=T_{1}+T_{2}$. Hence, we have $\mathscr{F} \in \mathfrak{B}$.

Let $\mathscr{F}$ be a sheaf of type (B), that is, it corresponds to a pair of two curves $\left\{T_{1}, T_{2}\right\}$. Let us assume that $\mathscr{F}$ admits another sequence

$$
0 \longrightarrow \mathcal{O}_{T_{3}} \longrightarrow \mathscr{F} \longrightarrow \mathcal{O}_{T_{4}} \longrightarrow 0
$$

with $T_{3}, T_{4} \in\left|\mathcal{O}_{\mathrm{Q}}(1,1)\right|$. Note that $\mathcal{O}_{T_{i}}$ is stable for all $i$. Thus, the composite map $s: \mathcal{O}_{T_{3}} \rightarrow \mathscr{F} \rightarrow \mathcal{O}_{T_{2}}$ is either a zero map or an isomorphism. In the former case, we have $\mathcal{O}_{T_{3}} \cong \mathcal{O}_{T_{1}}$ and so $\mathcal{O}_{T_{4}} \cong \mathcal{O}_{T_{2}}$. In the latter case, we have $\mathcal{O}_{T_{3}} \cong \mathcal{O}_{T_{2}}$ and $\mathcal{O}_{T_{4}} \cong \mathcal{O}_{T_{1}}$. Hence, the class of a strictly semistable sheaf $\mathscr{F}$ corresponds to a uniquely determined pair of two curves in $\left|\mathscr{O}_{Q}(1,1)\right|$ and we have $\mathfrak{B} \cong\left(\mathbb{P}^{3} \times \mathbb{P}^{3}\right) / \mathbb{S}_{2}$. The second assertion follows from the fact that any symmetric product $S^{d}\left(\mathbb{P}^{N}\right)$ of any projective space is a rational variety (see Theorems 4.2 .8 and $4.2 .8^{\prime}$ in page 137 of $[11,12]$ ). 
Lemma 24. For two curves $T_{1}, T_{2} \in\left|\mathcal{O}_{\mathrm{Q}}(1,1)\right|$, one has

$$
\operatorname{dim} \operatorname{Ext}^{1}\left(\mathcal{O}_{T_{2}}, \mathcal{O}_{T_{1}}\right)= \begin{cases}3, & \text { if } T_{1}=T_{2} \\ 2, & \text { if } T_{1} \neq T_{2}\end{cases}
$$

Proof. Note that we have

$$
\operatorname{Ext}^{2}\left(\mathcal{O}_{T_{2}}, \mathcal{O}_{Q}(-1,-1)\right)=H^{0}\left(\mathcal{O}_{T_{2}}(-1,-1)\right)^{\vee}=0 .
$$

Thus, if we apply the functor $\operatorname{Hom}\left(\mathcal{O}_{T_{2}},-\right)$ to the sequence of $T_{1}$, we obtain

$$
\begin{gathered}
0 \longrightarrow \operatorname{Hom}\left(\mathcal{O}_{T_{2}}, \mathcal{O}_{T_{1}}\right) \longrightarrow \operatorname{Ext}^{1}\left(\mathcal{O}_{T_{2}}, \mathcal{O}_{Q}(-1,-1)\right) \\
\longrightarrow \operatorname{Ext}^{1}\left(\mathcal{O}_{T_{2}}, \mathcal{O}_{\mathrm{Q}}\right) \longrightarrow \operatorname{Ext}^{1}\left(\mathcal{O}_{T_{2}}, \mathcal{O}_{T_{1}}\right) \longrightarrow 0
\end{gathered}
$$

We also have

$$
\begin{gathered}
\operatorname{Ext}^{1}\left(\mathcal{O}_{T_{2}}, \mathcal{O}_{\mathrm{Q}}(-1,-1)\right) \cong H^{1}\left(\mathcal{O}_{T_{2}}(-1,-1)\right)^{\vee} \cong H^{0}\left(\mathcal{O}_{T_{2}}\right) \\
\operatorname{Ext}^{1}\left(\mathcal{O}_{T_{2}}, \mathcal{O}_{\mathrm{Q}}\right) \cong H^{1}\left(\mathcal{O}_{T_{2}}(-2,-2)\right)^{\vee} \cong H^{0}\left(\mathcal{O}_{T_{2}}(1,1)\right)
\end{gathered}
$$

and so their dimensions are 1 and 3 , respectively. As $\mathcal{O}_{\mathrm{Q}^{-}}$ sheaves, we have

$$
h^{0}\left(\mathscr{H} \text { om }\left(\mathcal{O}_{T_{2}}, \mathcal{O}_{T_{1}}\right)\right)= \begin{cases}1, & \text { if } T_{1}=T_{2} ; \\ 0, & \text { otherwise }\end{cases}
$$

for example, because $T_{1}$ and $T_{2}$ are reduced, and so the assertion is derived.

Lemma 25. The sheaves $\mathscr{F}$ of type $(C)$, but not of type $(B)$, are stable. In particular, the sheaves of type $(C)$ are semistable.

Proof. As before let us assume the existence of a proper subsheaf $\mathscr{K}$ of $\mathscr{F}$ with $p(\mathscr{K}) \geq 1 / 2$ and the quotient sheaf $\mathscr{H}:=\mathscr{F} / \mathscr{K}$. Since the composite $s: \mathscr{K} \hookrightarrow \mathscr{F}^{2} \rightarrow \mathcal{O}_{T_{2}}$ is not a zero map, thus we have $\operatorname{Im}(s) \cong \mathcal{O}_{T_{2}}(-Z)$ for a 0 dimensional subscheme $Z$ of $T_{2}$ with length $k$. In particular, its Hilbert bipolynomial is $y+1-k$. If we let $\chi_{\mathscr{K}}(x, y)=$ $m^{\prime} x+n^{\prime} y+t^{\prime}$, then we have $p(\mathscr{K})=t^{\prime} /\left(m^{\prime}+n^{\prime}\right) \geq 1 / 2$. In particular, we have $t^{\prime} \geq 1$. If we define $\mathscr{K}^{\prime}$ to be the kernel of the map $s$, then it is a subsheaf of $\mathcal{O}_{T_{1}}$ and thus we have $p\left(\mathscr{K}^{\prime}\right)=\left(t^{\prime}-1+k\right) /\left(m^{\prime}+n^{\prime}-1\right) \leq 1 / 3$. Combining the two inequalities, we have $k=0$ and so the map $s$ is surjective. Thus, we have $\mathscr{H} \cong \mathcal{O}_{T_{1}} / \mathscr{K}^{\prime}$. Note also that $t^{\prime}$ can be either 1 or 2 . If $t^{\prime}=2$, then we have $m^{\prime}=n^{\prime}=2$ and so $\chi_{\mathscr{K}^{\prime}}(x, y)=2 x+y+1=\chi_{\mathcal{O}_{T_{1}}}(x, y)$. In particular, we have $\mathscr{H}=0$ and so $\mathscr{K} \cong \mathscr{F}$, a contradiction. Now, assume $t^{\prime}=1$ and so $m^{\prime}+n^{\prime} \leq 2$. In particular, $\mathscr{H}$ is not a 0 dimensional sheaf. Moreover, $\mathscr{H}$ is a quotient sheaf of $\mathscr{O}_{T_{1}}$ with constant term 1 and so we have $\mathscr{H} \cong \mathcal{O}_{T_{3}}$ with $T_{3} \subset T_{1}$ and $T_{3} \in\left|\mathcal{O}_{\mathrm{Q}}(1,1)\right|$. For example, if $T_{3}=T_{1}$, then we have $\mathscr{K}^{\prime}=0$ and it contradicts the nontriviality of the extension $\mathscr{F}$. Thus, $\mathscr{F}$ also admits the sequence

$$
0 \longrightarrow \mathscr{K} \longrightarrow \mathscr{F} \longrightarrow \mathcal{O}_{T_{3}} \longrightarrow 0
$$

where $\chi_{\mathscr{K}}(x, y)=x+y+1$. Since $\mathscr{K}^{\prime}$ is a subsheaf of $\mathcal{O}_{T_{1}}$ with $\chi_{\mathscr{K}^{\prime}}(x, y)=x$, we have $\mathscr{K}^{\prime} \cong \mathcal{O}_{T_{4}}(-1,0)$, where $T_{4}$ is a subcurve of $T_{1}$ such that $T_{1}=T_{3}+T_{4}$. Thus, $\mathscr{K}$ is an extension of $\mathcal{O}_{T_{2}}$ by $\mathcal{O}_{T_{4}}(-1,0)$. It is nontrivial, otherwise we would have $\mathcal{O}_{T_{2}}$ as a direct factor of $\mathscr{F}$. Since there exists such a unique extension $\mathcal{O}_{T_{2}+T_{4}}, \mathscr{F}$ admits an extension of $\mathcal{O}_{T_{3}}$ by $\mathcal{O}_{T_{2}+T_{4}}$ :

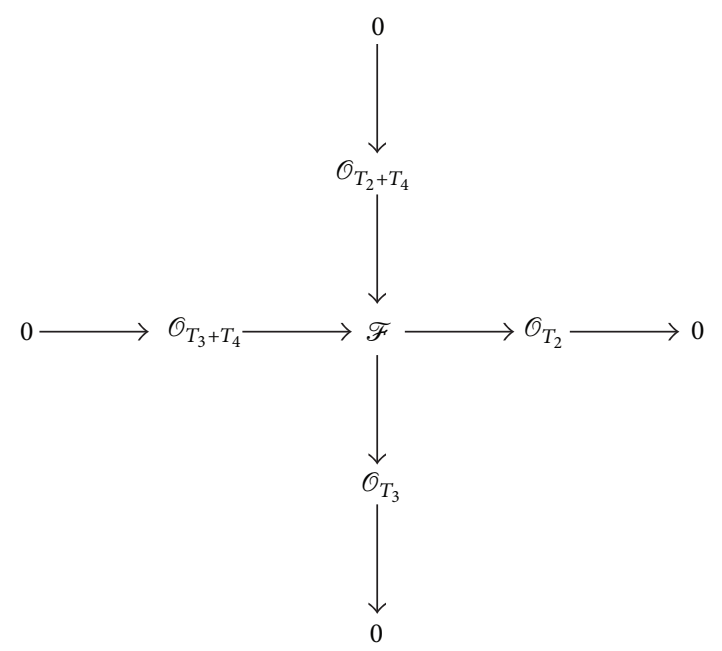

It implies that $\mathscr{F}$ is of type (B).

Lemma 26. Let $\mathscr{F}$ be a line bundle on a reduced curve $C \in$ $\left|\mathcal{O}_{\mathrm{Q}}(2,2)\right|$ with degree 2.

(1) $\mathscr{F}$ is semistable if and only if one has:

(a) $\operatorname{deg}\left(\left.\mathscr{F}\right|_{T}\right) \geq 1$ for all subcurves $T$ of $C$ in $\left|\mathcal{O}_{\mathrm{Q}}(a, b)\right|$ with $(0,0) \lesseqgtr(a, b) \lesseqgtr(1,1)$,

(b) $\operatorname{deg}\left(\left.\mathscr{F}\right|_{A}\right) \geq 0$ for each smooth subcurve $A$ of $C$ in $\left|\mathcal{O}_{\mathrm{Q}}(a, b)\right|$ with $(1,1) \leq(a, b) \lesseqgtr(2,2)$.

(2) $\mathscr{F}$ is stable if and only if $\operatorname{deg}\left(\left.\mathscr{F}\right|_{T}\right) \geq 1$ for all subcurves $T$ of $C$ in $\left|\mathscr{O}_{Q}(u, v)\right|$ with $(0,0) \lesseqgtr(u, v) \lessgtr(2,2)$.

Proof. In both parts, the "only if" part is obvious. Assume that $\mathscr{F}$ is not stable (resp., semistable) and take a proper subsheaf $\mathscr{H}$ of $\mathscr{F}$ with $p(\mathscr{H}) \geq p(\mathscr{F})($ resp. $p(\mathscr{H})>p(\mathscr{F}))$. Taking a saturation of $\mathscr{H}$ in $\mathscr{F}$, we may assume that $\mathscr{G}:=\mathscr{F} / \mathscr{H}$ is a pure sheaf. Call $A$ the scheme support of $\mathscr{H}$ and $T$ the scheme support of $\mathscr{G}$. The definition of scheme support of a purely 1 -dimensional sheaf gives $A+T=C$ as effective divisors. Thus $T$ has one of the types in the assertion. Since $C$ is reduced and $\mathscr{F}$ is a line bundle on $C$, the support of $\mathscr{G}$ must be a proper subcurve $T$ of $C$. If $T$ does not have a type of $\mathcal{O}_{Q}(1,2)$ or $\mathcal{O}_{Q}(2,1)$, then we are done. But the case of $T$ having such types is excluded using the argument in the proof of Lemma 18.

\section{Lemma 27. One has $\mathfrak{B} \cap \mathfrak{E} \neq \emptyset$.}

Proof. Let us set $B=B^{\prime}+T_{2}$ with $B^{\prime} \in\left|\mathcal{O}_{\mathrm{Q}}(0,1)\right|$ and $T_{2} \in\left|\mathcal{O}_{Q}(1,0)\right|$, and set $A \in\left|\mathcal{O}_{Q}(1,1)\right|$ to be smooth. For any extension $\mathscr{F} \in \mathfrak{B}$ of $\mathcal{O}_{B}$ by $O_{A}$, for example, $\mathscr{F}=\mathcal{O}_{A} \oplus \mathscr{O}_{B}$, let $\mathscr{H}$ be the kernel of the composition $\mathscr{F} \rightarrow \mathscr{O}_{B} \rightarrow \mathscr{O}_{T_{2}}$ and 
then $\mathscr{H}$ is a pure sheaf with $T_{1}:=A+B^{\prime}$ as its scheme support and has Hilbert bipolynomial $\chi_{\mathscr{H}}=\chi_{\mathcal{O}_{T_{1}}}$. Note that it has $\mathcal{O}_{A}$ as its subsheaf.

To prove $\mathscr{H} \cong \mathcal{O}_{T_{1}}$, it is sufficient to prove that $\mathscr{H}$ is semistable. Suppose $\mathscr{H}^{1}$ is not semistable and take a proper saturated stable subsheaf $\mathscr{G} \subset \mathscr{H}$ with $\chi_{\mathscr{G}}=a x+b y+$ $c$. Its scheme support is contained in $T_{1}$ and it is of type $(b, a)$. Without loss of generality, let us assume that $a \leq b$. First, assume $(a, b)=(1,2)$. In this case, we would have $c \geq 2$ because $p(\mathscr{G})>p(\mathscr{H})$ and so we have $h^{0}(\mathscr{H}) \geq 2$, contradicting the fact that $h^{0}(\mathscr{F})=2$ and that $\mathscr{F}$ is globally generated. Assume $a=b=1$. The map $\mathscr{G} \rightarrow \mathscr{H}$ on $A \backslash T_{2}$ must be just the inclusion $\mathscr{O}_{A} \rightarrow \mathscr{H}$, because $\left.\mathscr{H}\right|_{A \backslash T_{2}}$ is a line bundle. Thus either we have $\mathscr{G}=\mathcal{O}_{A}$ or $\mathcal{O}_{A}$ is not saturated in $\mathscr{H}$. Hence the saturation $\mathscr{A}$ of $\mathscr{O}_{A}$ in $\mathscr{F}$ has slope greater than $1 / 2$, contradicting the semistability of $\mathscr{F}$. Now assume $a=0$ and $b=1$, that is, $C_{\mathscr{G}}=B^{\prime}$. Since $B^{\prime}$ is smooth, $\mathscr{G}$ is a line bundle on $B^{\prime}$. If its degree $d$ is at least 1 , then $\mathscr{G}$ contradicts the semistability of $\mathscr{F}$. If $d \leq 0$, then we have $p(\mathscr{G})<p(\mathscr{H})$, a contradiction. Hence $\mathscr{F}$ is also contained in $\mathfrak{C}$.

Lemma 28. For $T_{1} \in\left|\mathcal{O}_{\mathrm{Q}}(1,2)\right|$ and $T_{2} \in\left|\mathcal{O}_{\mathrm{Q}}(1,0)\right|$, one has

$$
\operatorname{dim} \operatorname{Ext}^{1}\left(\mathcal{O}_{T_{2}}, \mathcal{O}_{T_{1}}\right)=2
$$

Proof. Applying the functor $\operatorname{Hom}\left(\mathcal{O}_{T_{2}},-\right)$ to the sequence of $T_{1}$, we obtain

$$
\begin{gathered}
0 \longrightarrow \operatorname{Ext}^{1}\left(\mathcal{O}_{T_{2}}, \mathcal{O}_{\mathrm{Q}}\right) \longrightarrow \operatorname{Ext}^{1}\left(\mathcal{O}_{T_{2}}, \mathcal{O}_{T_{1}}\right) \\
\longrightarrow \operatorname{Ext}^{1}\left(\mathcal{O}_{T_{2}}, \mathcal{O}_{\mathrm{Q}}(-1,-2)\right) \longrightarrow 0,
\end{gathered}
$$

since we have

$$
\begin{aligned}
\operatorname{Ext}^{1}\left(\mathcal{O}_{T_{2}}, \mathcal{O}_{Q}(-1,-2)\right) & \cong H^{1}\left(\mathcal{O}_{T_{2}}(-1,0)\right)^{\vee} \\
& \cong H^{1}\left(\mathcal{O}_{T_{2}}\right)^{\vee} \cong H^{0}\left(\mathcal{O}_{T_{2}}(-2)\right)=0
\end{aligned}
$$

and similarly $\operatorname{Ext}^{2}\left(\mathcal{O}_{T_{2}}, \mathcal{O}_{Q}\right)=0$. Note also that $\operatorname{Ext}^{1}\left(\mathcal{O}_{T_{2}}, \mathcal{O}_{\mathrm{Q}}\right) \cong H^{0}\left(\mathcal{O}_{T_{2}}\right)$ and $\operatorname{Ext}^{2}\left(\mathcal{O}_{T_{2}}, \mathcal{O}_{\mathrm{Q}}(-1,-2)\right) \cong$ $H^{0}\left(\mathcal{O}_{T_{2}}\right)^{\vee}$. Thus we have the assertion.

Remark 29. When $T_{1}$ and $T_{2}$ meet transversally at two points, say $P_{1}$ and $P_{2}$, then $\operatorname{Ext}^{1}\left(\mathcal{O}_{T_{2}}, \mathcal{O}_{T_{1}}\right)$ is the global sheaf of a sheaf with support on $P_{1}$ and $P_{2}$ with one copy of $\mathbb{C}$ on each point $P_{1}, P_{2}$,

$$
\operatorname{Ext}^{1}\left(\mathcal{O}_{T_{2}}, \mathcal{O}_{T_{1}}\right) \cong \mathbb{C}_{P_{1}} \oplus \mathbb{C}_{P_{2}}
$$

for the following reason.

Let $R$ be a regular local ring of dimension 2 and take $x, y$ generators of its maximal ideal. All $\mathrm{Ext}^{i}$ groups are with respect to $R$. Since $R /(y)$ is Gorenstein, so the duality gives $\operatorname{Ext}_{R}^{1}(R /(y), R) \cong R /(y)$ and $\operatorname{Ext}_{R}^{i}(R /(y), R)=0$ for all $i \neq 1$. From the exact sequence

$$
0 \longrightarrow R \stackrel{u}{\longrightarrow} R \longrightarrow \frac{R}{(x)} \longrightarrow 0,
$$

in which $u$ is the multiplication by $x$, we get that $\operatorname{Ext}_{R}^{1}(R /(y), R /(x))$ is the cokernel of the multiplication by $x$ in $R /(y) \rightarrow \mathrm{R} /(y)$; that is, we have $\operatorname{Ext}_{R}^{1}(R /(y), R /(x))=\mathbb{C}$. The same is true for extensions of $\mathcal{O}_{B}$ by $\mathcal{O}_{A}$ when $A$ and $B$ are transversal.

Lemma 30. Let $\mathscr{F}$ be a sheaf of type $(A)$ with no 0 dimensional torsion. Then $\mathscr{F}$ is semistable unless it admits the sequence

$$
0 \longrightarrow \mathcal{O}_{T_{2}} \longrightarrow \mathscr{F} \longrightarrow \mathcal{O}_{T_{1}} \longrightarrow 0
$$

where $T_{1} \in\left|\mathcal{O}_{\mathrm{Q}}(a, b)\right|$ and $T_{2} \in\left|\mathcal{O}_{\mathrm{Q}}(2-a, 2-b)\right|$ with $(a, b) \in$ $\{(1,2),(2,1)\}$.

Proof. Let $\mathscr{K}$ be a subsheaf $\mathscr{F}$ with maximal $p$-slope $p(\mathscr{K})>$ $1 / 2$ and so the quotient sheaf $\mathscr{H}:=\mathscr{F} / \mathscr{K}$ has no 0 dimensional torsion. Let us set $\chi_{\mathscr{K}}(x, y)=m^{\prime} x+n^{\prime} y+t^{\prime}$ with $t^{\prime} \geq 1$ and $(0,0) \lesseqgtr\left(m^{\prime}, n^{\prime}\right)$. If the composite $s: \mathscr{K} \hookrightarrow \mathscr{F}^{2} \rightarrow$ $\eta$ is a zero map, then $\mathscr{K}$ is a subsheaf destabilizing $\mathscr{O}_{C}$, a contradiction. If $s$ is not surjective, for instance, $\operatorname{Im}(s)=\mathscr{O}_{P} \varsubsetneqq$ $\eta$, then $\operatorname{ker}(s)$ is a subsheaf of $\mathcal{O}_{C}$ with Hilbert bipolynomial $m^{\prime} x+n^{\prime} y+t^{\prime}-1$. Thus we have $t^{\prime}=1$ and the quotient $\mathscr{H}^{\prime}:=$ $\sigma_{C} / \mathscr{K}^{\prime}$ has Hilbert bipolynomial with zero constant term. Since $\mathscr{H}^{\prime}$ has no 0 -dimensional torsion, we have $\mathscr{H}^{\prime} \cong \mathcal{O}_{D}$ for a curve $D$ contained in $C$. But the Hilbert bipolynomial of $\mathcal{O}_{D}$ has nonzero constant term, a contradiction. Thus the map $s$ is surjective. Following the same argument before, we obtain that $t^{\prime}=1$ and $m^{\prime}+n^{\prime} \leq 1$. Without loss of generality, let us assume that $\left(m^{\prime}, n^{\prime}\right)=(0,1)$. Then we have $\chi_{\mathscr{H}}(x, y)=$ $2 x+y+1$ and thus we have $\mathscr{H} \cong \mathcal{O}_{T_{1}}$, where $T_{1}$ is a curve contained in $C_{\mathscr{F}}$ and $T_{1} \in\left|\mathcal{O}_{Q}(1,2)\right|$. Since $\mathscr{K}$ is a subsheaf of $\mathscr{F}$ with $\chi_{\mathscr{K}}(x, y)=y+1$, we have $\mathscr{K} \cong \mathcal{O}_{T_{2}}$ since $\mathscr{K}$ has no 0 dimensional torsion. Thus $\mathscr{F}$ fits into the sequence (68).

Remark 31. Applying the functor $\operatorname{Hom}(\eta,-)$ to the sequence of $C \in\left|\mathcal{O}_{\mathrm{Q}}(2,2)\right|$, we obtain

$$
\begin{aligned}
& 0 \longrightarrow \operatorname{Ext}^{1}\left(\eta, \mathcal{O}_{C}\right) \longrightarrow \operatorname{Ext}^{2}\left(\eta, \mathcal{O}_{Q}(-2,-2)\right) \\
& \stackrel{f}{\longrightarrow} \operatorname{Ext}^{2}\left(\eta, \mathcal{O}_{Q}\right) .
\end{aligned}
$$

Since the map $f$ is the dual of the map $\operatorname{Hom}\left(\mathcal{O}_{Q}(2,2), \eta\right) \rightarrow$ $\operatorname{Hom}\left(\mathcal{O}_{\mathrm{Q}}, \eta\right)$ given by the multiplication by the defining equation of $C$, the map $f$ is a zero map and thus we have $\operatorname{Ext}^{1}\left(\eta, \mathcal{O}_{C}\right) \cong H^{0}(\eta)^{\vee}$. In particular its dimension is 2 .

Lemma 32. Let $\mathscr{F}$ be a sheaf of type $(B)$ fitting into an exact sequence

$$
0 \longrightarrow \mathcal{O}_{T_{1}} \longrightarrow \mathscr{F} \longrightarrow \mathcal{O}_{T_{2}} \longrightarrow 0
$$

with $T_{1}, T_{2} \in\left|\mathcal{O}_{\mathrm{Q}}(1,1)\right|$. Then $\mathscr{F}$ is of type $(A)$ if and only if $T_{1}$ and $T_{2}$ have no common components; that is, $C_{\mathscr{F}}$ has no multiple component.

Proof. If $T_{1}$ and $T_{2}$ have a common component, say $T$, then $\mathscr{F}$ has rank 2 at the general point of $T$ and thus $\mathscr{F}$ is not of type (A). 
Conversely, assume that $T_{1} \cap T_{2}$ is finite. Since we have $h^{1}\left(\mathcal{O}_{T_{1}}\right)=0$, the sequence $(70)$ implies that $h^{0}(\mathscr{F})=2$ and $\mathscr{F}$ is globally generated. Let $\sigma$ be a general section of $\mathscr{F}$ and then it does not vanish at the general point of any of the components of $C_{\mathscr{F}}$. Since $C_{\mathscr{F}}$ is reduced, $\sigma$ induces an injective map $\mathscr{O}_{C_{\mathscr{F}}} \hookrightarrow \mathscr{F}$ and thus $\mathscr{F}$ has type (A).

Lemma 33. Let $\mathscr{F}$ be a sheaf of type $(C)$.

(1) If $T_{2}$ is not a component of $T_{1}$, then $\mathscr{F}$ is of type $(A)$.

(2) If $T_{2}$ is a double component of $C_{\mathscr{F}}$, that is, $T_{2} \subset T_{1}$, then it is not of type $(A)$.

Proof. Let us assume that $T_{2} \in\left|\mathcal{O}_{\mathrm{Q}}(1,0)\right|$.

(1) Since $T_{2}$ is not a component of $T_{1}, \mathscr{F}$ is a line bundle on $C=C_{\mathscr{F}}$ outside finitely many points of $C$. Moreover, it is not an $\mathcal{O}_{T_{1}}$-sheaf. Note that $\mathscr{F}$ is globally generated since $\mathcal{O}_{T_{1}}$ and $\mathcal{O}_{T_{2}}$ are globally generated with $h^{1}\left(\mathcal{O}_{T_{1}}\right)=0$. Thus, a general section of $\mathscr{F}$ does not vanish at a general point of $T_{2}$ and so it does not induce an injection $\mathcal{O}_{T_{1}} \hookrightarrow \mathscr{F}$. Hence, $\mathscr{F}$ fits into some sequence (46).

(2) Let us set $C=2 T_{2}+T_{3}$ and $T_{1}:=T_{2}+T_{3}$, where $T_{3} \in\left|\mathcal{O}_{\mathrm{Q}}(0,2)\right|$. Let $\Gamma$ be the projectivisation of $\operatorname{Ext}^{1}\left(\mathcal{O}_{T_{2}}, \mathcal{O}_{T_{1}}\right)$ and in particular we have $\Gamma \cong \mathbb{P}^{1}$ by Lemma 28 . We also know from Lemma 25 that any $e \in \Gamma$ gives a semistable sheaf. Such a sheaf has rank 2 at the points of $T_{2} \backslash\left(T_{2} \cap T_{3}\right)$ and, in particular, it is not a line bundle over its support at a general point of $T_{2}$. Thus, it never fits into an exact sequence (46). Otherwise it would be locally free of rank 1 at each point of the support of $T_{3}$ but not in $T_{2}$.

In general, the question whether the variety $\mathscr{P}_{i c_{(m, n)}^{d}}^{d}$. We observed that $\mathbf{M}_{1}$ is rational and so is $\mathscr{P} i c_{(2,2)}^{1}$. Below we give a partial answer to this question in the case of $\mathscr{P}_{i c_{(2,2)}^{2}}^{2}$.

Theorem 34. $\mathbf{M}_{2}$ is unirational with degree 4.

Proof. Let us fix a smooth curve $C$ of bidegree $(2,2)$ in $Q$ and a point $P \in C$ to consider a sheaf $\mathscr{O}_{C}(P) \in \mathbf{M}_{1}$. If $\mathbb{T}_{P}$ is the tangent plane of $Q$ at $P$, then we have $\mathbb{T}_{P} \cap C=\left\{2 P, Q_{1}, Q_{2}\right\}$ for some points $Q_{1}, Q_{2}$ on $Q$ since $\operatorname{deg}(C)=4$. It defines a rational map

$$
\Phi: \mathbf{M}_{1}-\rightarrow \mathbf{M}_{2}
$$

sending $\mathcal{O}_{C}(P)$ to $\mathcal{O}_{C}\left(Q_{1}+Q_{2}\right)$. Note that $\mathcal{O}_{C}\left(Q_{1}+Q_{2}\right)=$ $\mathcal{O}_{C}(1,1)(-2 P)$. We claim that the map $\Phi$ is generically 4 to 1 and so the assertion follows.

Let $U$ (resp., $V$ ) be the dense open subset of $\mathbf{M}_{1}$ (resp., $\mathscr{A} \subset \mathbf{M}_{2}$ ) formed by the sheaves $\mathscr{F}$ such that $C_{\mathscr{F}}$ is smooth. Each element of $U$ (resp., $V$ ) is uniquely determined by a smooth $C \in\left|\mathcal{O}_{Q}(2,2)\right|$ and a degree one (resp., degree two) line bundle on $C$. By Riemann-Roch, each degree one line bundle on $C$ is associated with a unique $P \in C$. Then the map $\Phi$ sends $\mathscr{O}_{C}(P)$ to $\mathscr{R}:=\mathscr{O}_{C}(1,1)(-2 P)$. Fix any degree two line bundle $\mathscr{M}$ on $C$. Since we are in characteristic zero, there are exactly four line bundles $\mathscr{A}$ on $C$ such that $\mathscr{A}^{\otimes 2} \cong \mathcal{O}_{C}$. Hence, for each $\mathscr{R} \in \mathrm{Pic}^{2}(C)$ there are exactly 4 points $P \in C$ such that $\mathscr{R} \cong \mathcal{O}_{C}(1,1)(-2 P)$. Hence, $\Phi$ is dominant and the preimage of each element of $V$ has cardinality 4 .

We did not succeed in getting any smaller degree of unirationality of $\mathbf{M}_{2}$ as of now, and we left the rationality question as a conjecture.

Conjecture 35. $\mathbf{M}_{2}$ is rational.

\section{Conflict of Interests}

The authors declare that there is no conflict of interests regarding the publication of this paper.

\section{Acknowledgment}

Sukmoon Huh is supported by Basic Science Research Program 2010-0009195 through NRF funded by MEST.

\section{References}

[1] C. T. Simpson, "Moduli of representations of the fundamental group of a smooth projective variety I," Publications Mathématiques de L'Institut des Hautes Scientifiques, vol. 79, no. 1, pp. 47-129, 1994.

[2] H. G. Freiermuth, "On the moduli space $M_{P}\left(\mathbb{P}_{3}\right)$ of semi-stable sheaves on $\mathbb{P}_{3}$ with Hilbert polynomial $P(m)=3 m+1$," Diplomarbeit at University Kaiserlautern, 2000.

[3] H. G. Freiermuth and G. Trautmann, "On the moduli scheme of stable sheaves supported on cubic space curves," The American Journal of Mathematics, vol. 126, no. 2, pp. 363-393, 2004.

[4] M. Maican, "A duality result for moduli spaces of semistable sheaves supported on projective curves," Rendiconti del Seminario Matematico dell Università di Padova, vol. 123, pp. 55-68, 2010.

[5] J. Choi and K. Chung, "Cohomology bounds for sheavesof dimension one," http://arxiv.org/abs/1302.3691v2.

[6] J. le Potier, "Faisceaux semi-stables de dimension 1 sur le plan projectif," Revue Roumaine de Mathématique Pures et Appliquées, vol. 45, pp. 635-678, 1993.

[7] D. Eisenbud, Commutative Algebra: With a View Toward Algebraic Geometry, vol. 150 of Graduate Texts in Mathematics, Springer, New York, NY, USA, 1995.

[8] J. le Potier, "Faisceaux semi-stables et systemes coherents, Vector bundles in algebraic geometry," London Mathematical Society, vol. 208, pp. 179-240, 1995.

[9] D. Huybrechts and M. Lehn, The Geometry of Moduli Spaces of Sheaves, vol. E31 of Aspects of Mathematics, Vieweg, 1997.

[10] E. Ballico and F. Malaspina, "Regularity and cohomological splitting conditions for vector bundles on multiprojective spaces," Journal of Algebra, vol. 345, no. 1, pp. 137-149, 2011.

[11] I. M. Gel'fand, M. M. Kapranov, and A. V. Zelevinsky, Discriminants, Resultants, and Multidimensional Determinants, Mathematics: Theory \& Applications, Birkhäuser, Boston, Mass, USA, 1994

[12] A. Mattuck, “The field of multisymmetric functions," Proceedings of the American Mathematical Society, vol. 19, pp. 764-765, 1968. 


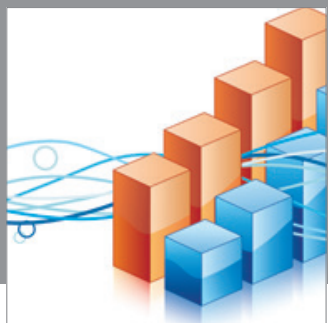

Advances in

Operations Research

mansans

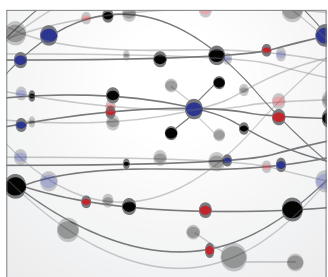

The Scientific World Journal
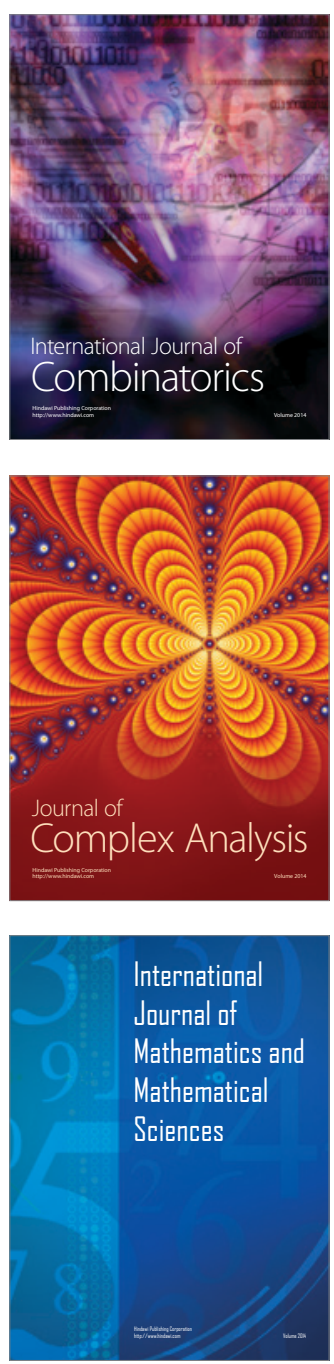
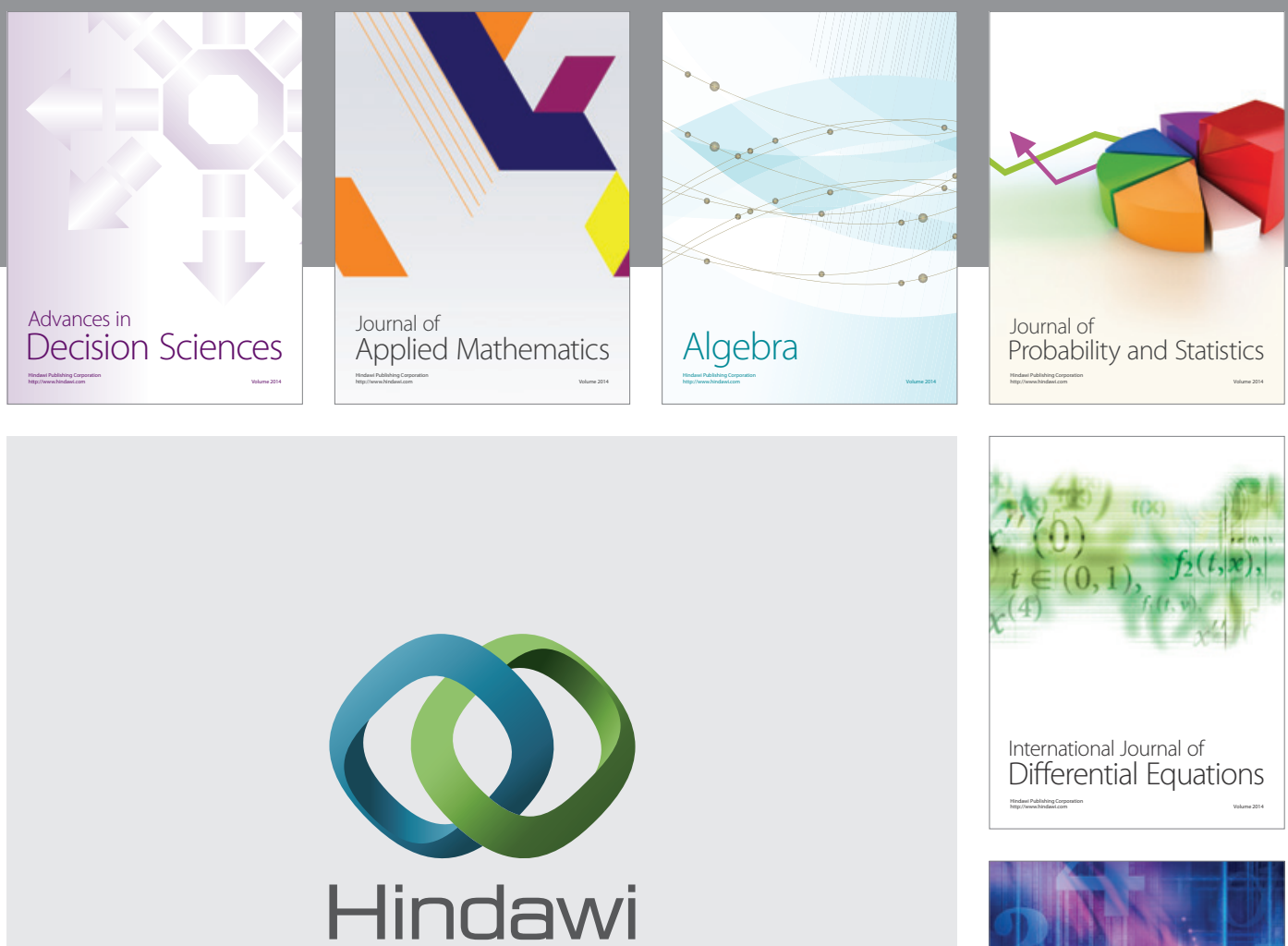

Submit your manuscripts at http://www.hindawi.com
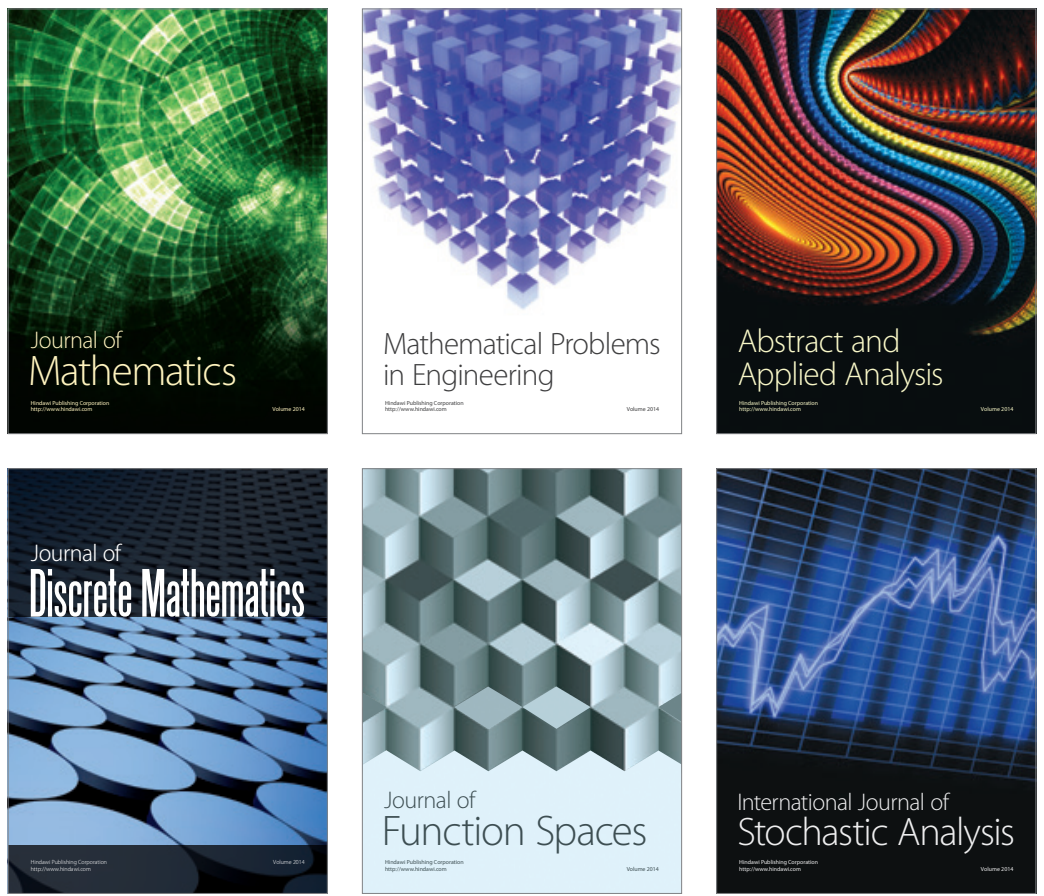

Journal of

Function Spaces

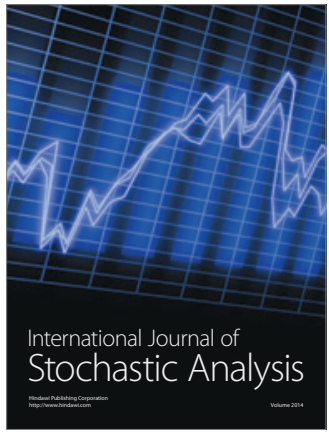

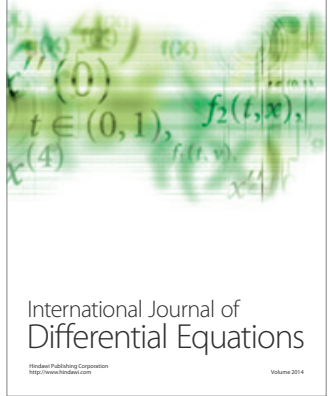
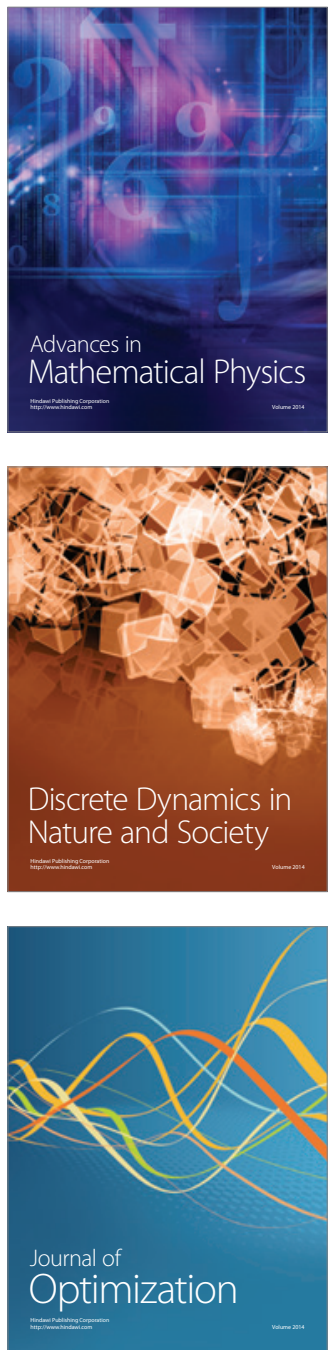